\title{
Construction of magnetic-carbon-quantum- dots-probe-labeled apoferritin nanocages for bioimaging and targeted therapy
}

This article was published in the following Dove Press journal:

International Journal of Nanomedicine

6 September 2016

Number of times this article has been viewed

\author{
Hanchun Yao ${ }^{1,2}$ \\ Li Su' \\ Man Zeng' \\ Li Cao' \\ Weiwei Zhao' \\ Chengqun Chen ${ }^{3}$ \\ Bin $\mathrm{Du}^{1,2}$ \\ Jie Zhou ${ }^{1,2}$
}

'School of Pharmaceutical Sciences, Zhengzhou University, ${ }^{2}$ Collaborative Innovation Center of Drug Research and Safety Evaluation, Henan Province, ${ }^{3}$ Department of Pharmacy, The First Affiliated Hospital of Zhengzhou University, Zhengzhou, People's

Republic of China
Correspondence: Bin Du; Jie Zhou School of Pharmaceutical Sciences, Zhengzhou University, No 100, Kexue Road, Zhengzhou 45000I, People's Republic of China

Tel +86 37| 6778 I902

Fax $+8637 \mid 67781908$

Email dubin@zzu.edu.cn; zhj_paper@sina.com

\begin{abstract}
Carbon dots (CDs) are one of the most highlighted carbon-based materials for biological applications, such as optical imaging nanoprobes, which are used for labeling cells in cancer treatment mainly due to their biocompatibility and unique optical properties. In this study, gadolinium (Gd)-complex-containing CDs were obtained through a one-step microwave method to develop multimodal nanoprobes integrating the advantages of optical and magnetic imaging. The obtained Gd-CDs exhibited highly fluorescent properties with excellent water solubility and biological compatibility. Natural apoferritin (AFn) nanocages, an excellent drug delivery carrier, are hollow in structure, with their $\mathrm{pH}$-dependent, unfolding-refolding process at $\mathrm{pH} 2.0$ and 7.4. The chemotherapeutic drug doxorubicin (DOX) can be highly effective and encapsulated into AFn cavity. A widely used tumor-targeting molecule, folic acid (FA), functionalized the surface of AFn to obtain an active tumor targeting effect on MCF-7 cells and malignant tumors in mice models. In this study, an AFn nanocarrier encapsulating high concentration of DOX labeled with magnetic and fluorescent Gd-CDs probe was developed. Gd-CDs exhibited a unique green photoluminescence and almost no toxicity compared with free $\mathrm{GdCl}_{3}$. Furthermore, Gd-doped CDs significantly increased the circulation time and decreased the toxicity of $\mathrm{Gd}^{3+}$ in in vitro and in vivo magnetic resonance imaging, which demonstrated that the AFn nanocages labeled with Gd-CD compounds could serve as an excellent $\mathrm{T}_{1}$ contrast agent for magnetic resonance imaging. The self-assembling multifunctional Gd-CDs/AFn (DOX)/FA nanoparticles have a great potential for cancer theranostic applications.
\end{abstract}

Keywords: Gd-CDs, fluorescence, magnetic, apoferritin nanocages, imaging

\section{Introduction}

Fluorescent nanoparticles including quantum dots (QDs) and organic dyes have been widely used for labeling cells and in imaging due to their outstanding photoluminescence (PL) properties. ${ }^{1,2}$ However, traditional QDs contain heavy metal elements; the cytotoxicity of the heavy metal ions in biological system and the potential environmental harmfulness limit further applications of QDs in theranostics. ${ }^{3,4}$ Conventional organic dyes have larger toxicity and poor water solubility and photostability. Compared to organic dyes, carbon dots (CDs) are superior due to their low cytotoxicity, excellent aqueous solubility, good biocompatibility, broader PL profiles, and high photostability. CDs have attracted tremendous attention in diverse research fields and have been synthesized and used in biochemical applications. ${ }^{5} \mathrm{CDs}$ were used as a new type of carbon nanomaterial because of their size being $<10 \mathrm{~nm} .{ }^{6,7}$ This together with the easy functionalization makes CDs optimal materials to fabricate bimodal imaging nanoprobes. 
Luminescent CDs were discovered through purification of crude carbon nanotubes in $2004 .{ }^{8}$ Subsequently, scientists began investigations on the preparation of CDs via various methods, such as arc discharge, laser ablation, electrochemical oxidation, combustion, thermal decomposition or oxidation, and microwave-assisted pyrolysis. ${ }^{9}$ Among the abovementioned approaches, microwave-assisted pyrolysis has been regarded as an economical and green method, which makes it useful for preparation of simultaneous, homogeneous, and uniform-sized CDs through fast heating. A one-step microwave-assisted polyol method was developed by Gong et al to prepare green-luminescent CDs, which could be efficiently taken up by the C6 cells..$^{10,11}$ In this study, gadolinium (Gd)-doped green-luminescent CDs were prepared as a novel bimodal nanoprobe by a onestep microwave-assisted method within few minutes. The obtained Gd-CDs could maintain long-lasting brightness and be easily functionalized with amino groups. As already known, Gd compounds, such as Gd(III)-diethylenetriami nepentaacetic acid ([Gd-DTPA $]^{-2}$ ), were used as clinical $\mathrm{T}_{1}$-shortening agents owing to the advantages of magnetic resonance imaging (MRI) contrast agents. ${ }^{12,13}$ Moreover, the potential of as-prepared Gd-CDs as a bimodal (MRI/optical) imaging nanoprobe was explored by examining their distinctive PL and magnetic properties.

Caged protein nanoparticles possess many desirable features for drug delivery, such as ideal sizes for endocytosis, nontoxic biodegradability, and the ability to functionalize at three distinct interfaces (external, internal, and inter-subunit) using the tools of protein engineering. ${ }^{14}$ Ferritin is a major iron storage protein in humans and most living organisms. The ferritin nanocage is composed of 24 subunits, which self-assemble to form a cage-like nanostructure. Apoferritin (AFn) was obtained from ferritin by removing iron ions from its internal core. When expressed artificially in iron-free conditions, the yielded AFn is hollow, comprising a cavity that can be loaded with different species..$^{15}$ The characteristics such as good biocompatibility, non-immunogenicity, and unique nanostructure were the important reasons for choosing ferritin nanocage as a drug carrier. It has been observed that in strong acidic environments ( $\mathrm{pH} 2.0)$, the ferritin cage disassembles into discrete subunits, while at $\mathrm{pH} 7.4$ these subunits once again self-assemble through non-covalent interactions and restore the cage-like structure. ${ }^{16}$ This unique $\mathrm{pH}-$ responsive architecture has rendered it especially convenient to build multifunctional hybrid ferritins by conjugating different small molecules onto the subunits. Ji et al used AFn as a carrier to encapsulate three platinum anticancer drugs, cisplatin, carboplatin, and oxaliplatin, into its cavity. ${ }^{17}$ Doxorubicin (DOX) is a kind of widely used antitumor antibiotic that could inhibit the synthesis of RNA and DNA in tumor cells. ${ }^{18}$ By increasing the $\mathrm{pH}$ of the solution, the AFn structure could be reassembled and DOX molecules could be encapsulated into the cavity. This feature allows the application of AFn as a drug nanocarrier with specific low $\mathrm{pH}$-initiated release. ${ }^{19}$ It is obvious that $\mathrm{AFn}$ (DOX) nanoparticles for tumor-specific drug delivery have an advantage over active pharmaceutical ingredients in tumor inhibition and in vivo toxicity. ${ }^{20}$ In this study, AFn (DOX) nanoparticles were labeled with fluorescent and magnetic Gd-CDs for bimodal (MRI/optical) imaging. The surface of Gd-CDs was made to connect to AFn nanocages through amidation process. Their combination efficiently decreased the toxicity of Gd-CDs and increased the solubility of Gd-CDs in aqueous solution. Folic acid (FA) was connected to the surface of AFn, which aimed at targeting tumors. AFn nanocarrier specifically delivered a high concentration of DOX to tumor cells and significantly inhibited tumor growth with a single-dose treatment; meanwhile, it also showed excellent biocompatibility and safety profiles in murine cancer models. The specific synthetic route of Gd-CDs/AFn (DOX)/FA is shown in Figure 1. AFn-enabled, $\mathrm{pH}$-triggered content release in combination with magnetic CDs provided the required properties and behavior of fluorescence and magnetism. As shown in this study, DOX can be effectively encapsulated into the AFn cavity and transported via FA to the site of action. The prepared Gd-CDs acted as a fluorescent probe for labeling AFn (DOX) nanoparticles, which have potential future applications in microscopy or whole-body small animal imaging. Moreover, the application of Gd-CDs/AFn (DOX)/FA in magnetic resonance (MR) contrast agent for in vivo MRI also was assessed in this study.

\section{Materials and methods \\ Materials}

DOX (purity $>98 \%$ ) was obtained from Beijing Yi-He Biotech Co., Ltd., Beijing, People's Republic of China. $\mathrm{GdCl}_{3}$ (purity $>99.9$, wt.\%) was purchased from Sinopharm Chemical Reagent Co., Ltd. (Shanghai, People's Republic of China). Ferritin from equine spleen, FA, citric acid, polyethylenimine (PEI; molecular weight $=2,500$ ), diethylene glycol, ethylenediamine, formamide, $\mathrm{N}$-hydroxysuccinimide (NHS), N-(3-dimethylaminopropyl-N'-ethylcarbodiimide) hydrochloride (EDC-HCl), and dimethyl sulfoxide were obtained from Sigma-Aldrich Co. (St Louis, MO, USA). 3-(4,5-Dimethylthiazolyl-2)-2,5-diphenyltetrazolium 


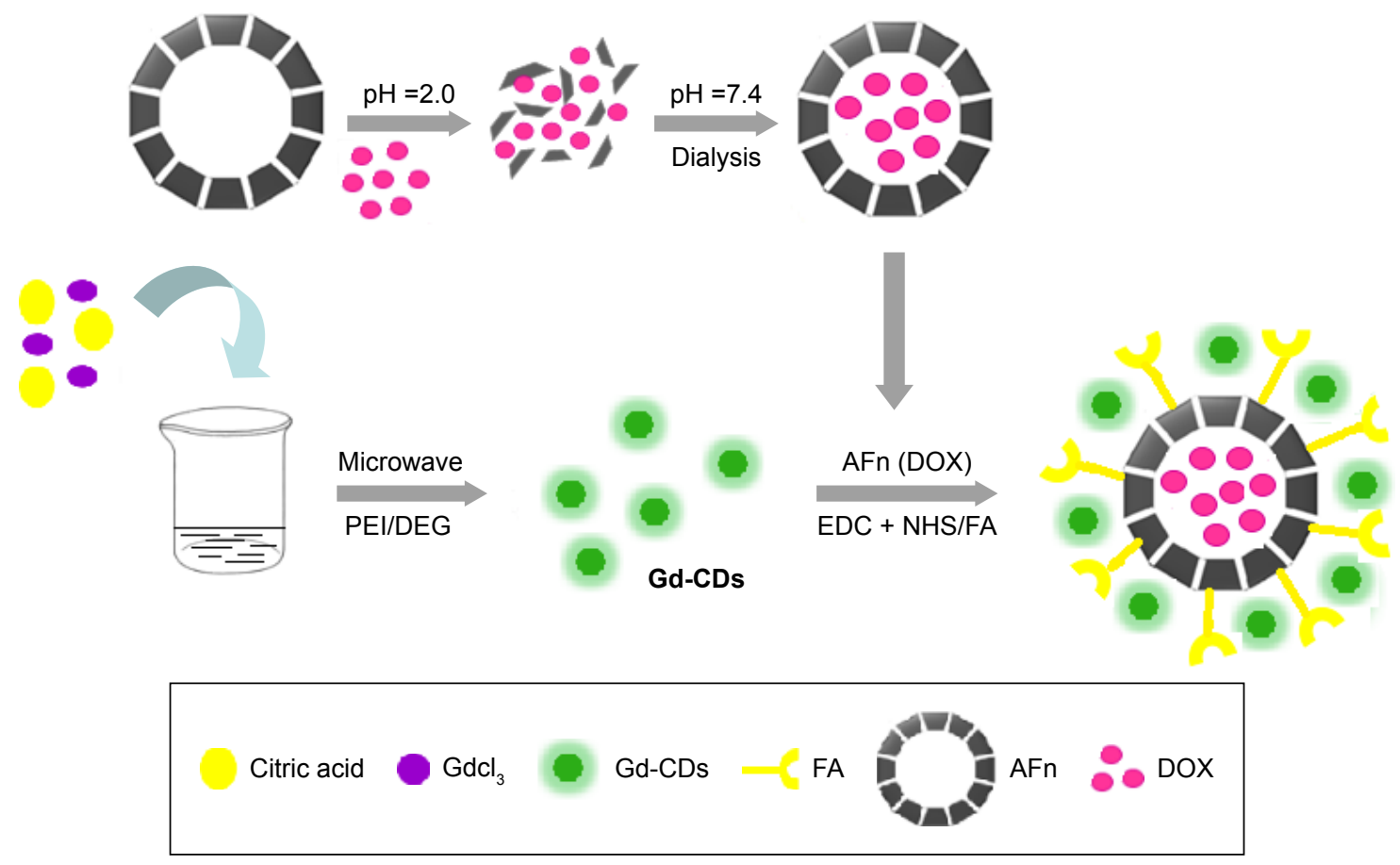

Figure I Illustration of Gd-CDs/AFn (DOX)/FA nanocomposite preparation.

Abbreviations: AFn, apoferritin; CDs, carbon dots; DEG, diethylene glycol; DOX, doxorubicin; EDC, N-(3-dimethylaminopropyl-N'-ethylcarbodiimide); FA, folic acid; Gd, gadolinium; NHS, N-hydroxysuccinimide; PEl, polyethylenimine.

bromide (MTT), Dulbecco's Modified Eagle's Medium cell culture medium, penicillin, streptomycin, fetal bovine serum, and heparin sodium were purchased from Thermo Fisher Scientific (Waltham, MA, USA). Other reagents were acquired from China National Medicines Corporation Ltd. The dialysis bags (molecular weight cutoff $[\mathrm{MWCO}]=10,000)$ were obtained from Spectrum Laboratories, Inc. Ultrapure water was used throughout the experiments.

\section{Preparation of luminescent Gd-CDs}

Gd-CDs were synthesized by a one-step microwave-assisted pyrolysis method. ${ }^{21}$ Briefly, citric acid ( $1 \mathrm{~g}$ ) and PEI (molecular weight $=2,500,0.25 \mathrm{~g}$ ) were dissolved in $10 \mathrm{~mL}$ glycerin; subsequently, $0.2 \mathrm{~g} \mathrm{GdCl}_{3}$ was added to the abovementioned solution. The mixture was sonicated to form a homogeneous solution, then placed in a domestic microwave, and heated for 5 minutes at $750 \mathrm{~W}$ power. In the reaction process, citric acid was carbonized into $\mathrm{CDs}$, and the surface of CDs was modified with PEI. Furthermore, $\mathrm{Gd}^{3+}$ was doped into CDs. After the mixed solution naturally cooled down to room temperature, the resultant solution turned dark brownish, indicating the formation of Gd-CDs. A yellow color pure solution of Gd-CDs was achieved after the mixed solution was purified by dialyzing against ultrapure water by using dialysis membranes with $\mathrm{MWCO}=1,000$. The collected Gd-CD solutions were dried in vacuum for 24 hours.

\section{Preparation of AFn}

Using the dialysis method, ${ }^{22}$ AFn was obtained by removing soluble iron from ferritin, according to the following steps: horse spleen ferritin samples were diluted 1,000 times and dialyzed in NaAc solution ( $0.1 \mathrm{M}, \mathrm{pH}$ 5.5) at low temperature. After concentrating to $300 \mu \mathrm{L}$, the ferritin sample was dialyzed in NaAc solution for 2 hours (0.1 M, pH 5.5), and $20 \mu \mathrm{L}$ mercaptoacetic acid (TGA) was added. Then, $10 \mu \mathrm{L}$ TGA was added and dialysis in NaAc solution was continued $(0.1 \mathrm{M}, \mathrm{pH} 5.5,250 \mathrm{~mL})$ for 1 hour. Finally, preliminary AFn sample was purified by dialyzing in NaAc solution $(0.1 \mathrm{M}, \mathrm{pH}$ $5.5,250 \mathrm{~mL}$ ) for 20 minutes after the replacement of dialysis fluid. The sample was concentrated with an MWCO of 10,000 ultrafiltration. Then, AFn solution was collected and stored at $4^{\circ} \mathrm{C}$ in the refrigerator. The obtained AFn sample was characterized by transmission electron microscope (TEM).

\section{Preparation of DOX-loaded AFn}

The loading of DOX into the cavity of AFn was carried out as per the following procedures. ${ }^{23}$ Briefly, $0.5 \mathrm{mg} / \mathrm{mL}$ of AFn was dissolved in $0.15 \mathrm{M} \mathrm{NaCl}$. Then, DOX was added into the solution at a final concentration of $1 \mathrm{mg} / \mathrm{mL}$ and gently stirred 
for 30 minutes at room temperature. The $\mathrm{pH}$ of the mixed solution was adjusted to 2.0 using $0.1 \mathrm{M} \mathrm{HCl}$ and then the $\mathrm{pH}$ value was maintained for $\sim 15$ minutes. Afterward, the $\mathrm{pH}$ was slowly increased up to 7.4 using $0.1 \mathrm{M} \mathrm{NaOH}$. The resulting solution was stirred at 1,000 rpm under room temperature for 2 hours. The excess DOX and the drug molecules outside of the protein shell were removed by dialyzing the solution against saline water $(0.15 \mathrm{M} \mathrm{NaCl}$, renewed three times $)$ for 24 hours through dialysis bags of MWCO 10,000.

\section{Attachment of Gd-CDs to AFn (DOX)}

PEI-coated Gd-CDs were terminated with amine groups. Covalent binding of AFn to the Gd-CDs was performed using a modification of the standard EDC-NHS reaction. Briefly, $1 \mathrm{mg}$ of AFn (DOX) was dissolved in $1 \mathrm{~mL}$ phosphate-buffered saline (PBS), followed by the addition of 1 molar equivalent of NHS and EDC for activation for 30 minutes. Following the activation of carboxyl groups, $2 \mathrm{mg}$ of Gd-CDs in PBS $(\mathrm{pH}=7.4,2 \mathrm{~mL})$ was added and the mixed solution was allowed to react at room temperature for 3 hours. The resultant mixture was purified through a dialysis membrane $(\mathrm{MWCO}=10,000)$ against distilled water for 48 hours. The products were dispersed in PBS for further characterization and application.

\section{Conjugation of FA to Gd-CDs/AFn (DOX)}

Activation of FA was carried out prior to attachment. Attachment was carried out by adding $8 \mathrm{~mL}$ of Gd-CDs/ AFn (DOX) complex solution to $2 \mathrm{~mL}$ of activated FA. The solution was subjected to purging under $\mathrm{N}_{2}$ atmosphere for 4 hours under stirring conditions. Both inlet and outlet valves were closed, and the reaction was allowed to take place for 24 hours. Finally, the solution was dialyzed by a membrane $(\mathrm{MWCO}=10,000)$ to remove free FA.

\section{Characterization}

Fourier transform infrared (FTIR) spectra were obtained on a Bruker Vector 22 spectrophotometer (Shimadzu Corp., Kyoto, Japan) in the range of 4,000-500 $\mathrm{cm}^{-1}$ and recorded on solid samples in a $\mathrm{KBr}$ matrix. Fluorescence spectra were obtained on an RF-5301 spectrofluorometer (Shimadzu Corp.) equipped with a $450 \mathrm{~W}$ Xenon lamp. Ultraviolet (UV)visible absorption spectra were measured using UV-visible spectrometer (Lambda 35; PerkinElmer Inc., Waltham, MA, USA). X-ray photoelectron spectroscopy (XPS) was obtained on an axis ultra-spectrometer (Kratos, Manchester, UK) by using mono-Al $\mathrm{K}$ a line $(1,486.71 \mathrm{eV})$ radiation at a power of 225 W. Dynamic light scattering (DLS; Zetasizer Nano ZS-90; Malvern Instruments, Malvern, UK) and TEM (Tecnai
G2 20; FEI, Hillsboro, OR, USA) were used for characterizing particle size, zeta potential, and morphology of Gd-CDs and Gd-CDs/AFn (DOX)/FA, respectively. The in vitro and in vivo $\mathrm{T}_{1}$-weighted $\mathrm{MR}$ images were conducted on a $3 \mathrm{~T}$ clinical MRI scanner (Siemens, Munich, Germany).

\section{Determination of entrapment efficiency}

After encapsulation, $5 \mathrm{~mL}$ of AFn (DOX) complex was dialyzed against nanopure water, and the concentration of unbound DOX was measured by UV spectrophotometry. A curve as plotted with the standard DOX concentration as $X$-axis and UV absorption intensity with linear regression as the $Y$-axis. The concentration of DOX was calculated by the DOX UV absorption standard curve (linear line equation: $y=3.7632 x$ ).

Encapsulation efficiency was calculated using the following equation:

$$
\mathrm{EE}(\%)=\frac{\text { Amount of DOX loaded in AFn }}{\text { Initial amount of the DOX }} \times 100
$$

\section{Drug release and kinetics of DOX}

To assay the drug release profile under physiological conditions, $2 \mathrm{~mL}$ of Gd-CDs/AFn (DOX)/FA solution was added into two dialysis bags, respectively, of MWCO 8,000-10,000. Then, the two dialysis bags were placed in $50 \mathrm{~mL}$ PBS solution ( $\mathrm{pH} 7.4$ ) and $50 \mathrm{~mL}$ of acetic acid buffer ( $\mathrm{pH}$ 5.5), respectively, and heated at $37^{\circ} \mathrm{C}$ under mild intermittent stirring. Original solution $(0.2 \mathrm{~mL})$ was collected at 0.5 hours, 1 hour, 2 hours, 4 hours, 8 hours, 12 hours, 36 hours, 48 hours, 60 hours, and 72 hours, while adding $0.2 \mathrm{~mL}$ PBS or acetic acid buffer into the original solution. The concentration of DOX was calculated using a standard calibration curve method, with UV absorption wavelength of $475 \mathrm{~nm}$. The DOX accumulation release was calculated at different time points.

\section{Cell imaging}

For fluorescence incubation imaging, MCF-7 cells were harvested by detachment using trypsin solution and then resuspended in fresh medium. The cells were plated in $35 \mathrm{~mm}$ glass-bottom plate containing the culture medium and incubated for 12 hours, with each well containing $1 \mathrm{~mL}$ of MCF-7 cells. ${ }^{24}$ Then, different concentrations of $2 \mathrm{~mL}$ sample solutions were added into the plate. After incubation for a certain time, the cells were washed several times with PBS solution to remove residual particles. Finally, the colocalization of Gd-CDs, Gd-CDs/AFn (DOX), and Gd-CDs/AFn (DOX)/FA with cellular structures was imaged using confocal laser scanning microscopy with excitation and emission filters of preconfigured settings. 


\section{In vitro cytotoxicity and inhibition assay}

The cytotoxicity effects of Gd-CDs and Gd-CDs/AFn complex and inhibition of free DOX, Gd-CDs/AFn (DOX), and Gd-CDs/AFn (DOX)/FA compositions toward MCF-7 cells were evaluated by a standard MTT assay. MCF-7 cells were added to 96-well culture plate. The concentration of inhibition was set at $0.5 \mu \mathrm{g} / \mathrm{mL}, 1.25 \mu \mathrm{g} / \mathrm{mL}, 2.5 \mu \mathrm{g} / \mathrm{mL}, 5 \mu \mathrm{g} / \mathrm{mL}$, and $10 \mu \mathrm{g} / \mathrm{mL}$, and the concentrations of Gd-CDs were $6 \mu \mathrm{g} / \mathrm{mL}$, $12 \mu \mathrm{g} / \mathrm{mL}, 25 \mu \mathrm{g} / \mathrm{mL}, 50 \mu \mathrm{g} / \mathrm{mL}, 100 \mu \mathrm{g} / \mathrm{mL}$, and $200 \mu \mathrm{g} / \mathrm{mL}$. The cell viabilities were equal to the average of the absorbance obtained after the experimental group was divided by the blank control group, then by the mean of the absorbance value of the control group, and finally by $100 \%$.

\section{In vivo antitumor effect}

Until the tumor volume reached $60-100 \mathrm{~mm}^{3}$, the healthy mice with similar tumor volume and weight were randomly assigned to one of the following five treatment groups ( $\mathrm{n}=5$ for per group): physiological saline group, free DOX, Gd-CDs/AFn, Gd-CDs/AFn (DOX), and Gd-CDs/AFn $(\mathrm{DOX}) / \mathrm{FA}$. In this experiment, DOX concentration was converted into the clinical dosage. Five groups of mice were administered via tail vein injection (once every 2 days, a total of seven times of administration), and all drugs were diluted with PBS and injected in a single dose. Body weight of all mice in the five groups was recorded every other day ${ }^{25}$ Simultaneously, the sarcoma of tumor-bearing mice, both long diameter $(a)$ and short diameter $(b)$, was measured using a digital Vernier caliper, according to the formula of tumor volume $V=1 / 2 \times a \times b^{2}$, and the tumor volume was recorded for each measurement. After 14 days of treatment, tumor-bearing mice were killed; the heart, liver, spleen, lung, kidney, and tumor tissues were taken out, put into $10 \%$ formalin solution, and paraffin embedded for hematoxylin and eosin staining. Morphological changes were observed and recorded using Leica TCS-SP5 confocal microscope with the supplied software (Leica 5 Confocal Software Version 2.0; Leica Microsystems, Wetzlar, Germany). All animalhandling procedures were performed according to a protocol approved by the Henan Province Laboratory Animals Center. All animal experiments were approved by the Experimental Animal Ethical Committee of Zhengzhou University.

\section{Pharmacokinetic study}

Gd-CDs/AFn (DOX)/FA (DOX dose: $5 \mathrm{mg} / \mathrm{kg}$ ) or free DOX was intravenously injected into tumor-free healthy C57 mice ( $\mathrm{n}=6$ for each group). Then, $0.5 \mathrm{~mL}$ blood was collected from the eyes at set time points, ie, 0.083 hours, 0.25 hours, 0.5 hours, 1 hour, 2 hours, 3 hours, 4 hours,
6 hours, 8 hours, 12 hours, and 24 hours. The obtained upper plasma samples were placed in $1 \mathrm{~mL}$ centrifuge tubes after the blood was separated, and then acetonitrile $(1 \mathrm{~mL})$ was added to the abovementioned tubes and centrifuged after mixing by vortex. The supernatant was collected and dried by airflow $\left(40^{\circ} \mathrm{C}\right)$, followed by addition of ethanol $(0.1 \mathrm{~mL})$ to dissolve DOX, and DOX in the blood samples was detected by high-performance liquid chromatography under the optimum conditions. The pharmacokinetic parameters were calculated using Kinetica 4.4.1 program (Thermo Fisher Scientific).

\section{In vitro/vivo MRI}

Gd-CDs/AFn (DOX)/FA of different solutions at $0.1 \mathrm{mg} / \mathrm{mL}$, $0.2 \mathrm{mg} / \mathrm{mL}, 0.4 \mathrm{mg} / \mathrm{mL}, 0.6 \mathrm{mg} / \mathrm{mL}$, and $0.8 \mathrm{mg} / \mathrm{mL}$ were dispersed in double-distilled water. Then, the relaxivity of Gd-CDs/AFn (DOX)/FA was obtained on a 3 T MRI instrument. For in vivo MRI, the tumor-bearing mice were intravenously injected with $0.2 \mathrm{~mL}$ of Gd-CDs/AFn (DOX)/ FA (Gd dose: $1 \mathrm{mM} / \mathrm{kg}$ ). Saline group was used as control. ${ }^{26}$ After injection at different time points, MRI was conducted on a $3 \mathrm{~T}$ clinical MRI scanner.

\section{Results and discussion Synthesis and characterization of Gd-CDs}

Citric acid is an important organic acid, which possesses good water solubility and is commonly used as a carbon source to synthesize CDs. It was previously reported that bare carbon QDs have faint luminescence and poor water solubility, while modified CDs show strong fluorescence properties and good biocompatibility. PEI with rich amino groups on the surface was used as a modified group. As a result, luminescent $\mathrm{CDs}$ were formed with the color of the CDs solution changing from colorless clarification to yellow brown after micro-assisted heating. In the meantime, Gd ion participated in the formation of CDs, and thus luminescent and magnetic Gd-CDs were achieved. The size and morphology of Gd-CDs were observed by microwave-assisted polyol method using high-resolution TEM (HRTEM). It can be seen from the HRTEM image that synthetic Gd-CDs were individually dispersed, as shown in the inset of Figure 2A, showing typical quasi-spherical nanodots with average diameters of $\sim 5 \mathrm{~nm}$. The polydispersity index of Gd-CDs was 0.201. Particle size distribution map (Figure 2B) shows that the aqueous dispersion of Gd-CDs had a narrow size distribution with diameters of $\sim 5 \mathrm{~nm}$, which is consistent with the abovementioned HRTEM image. 

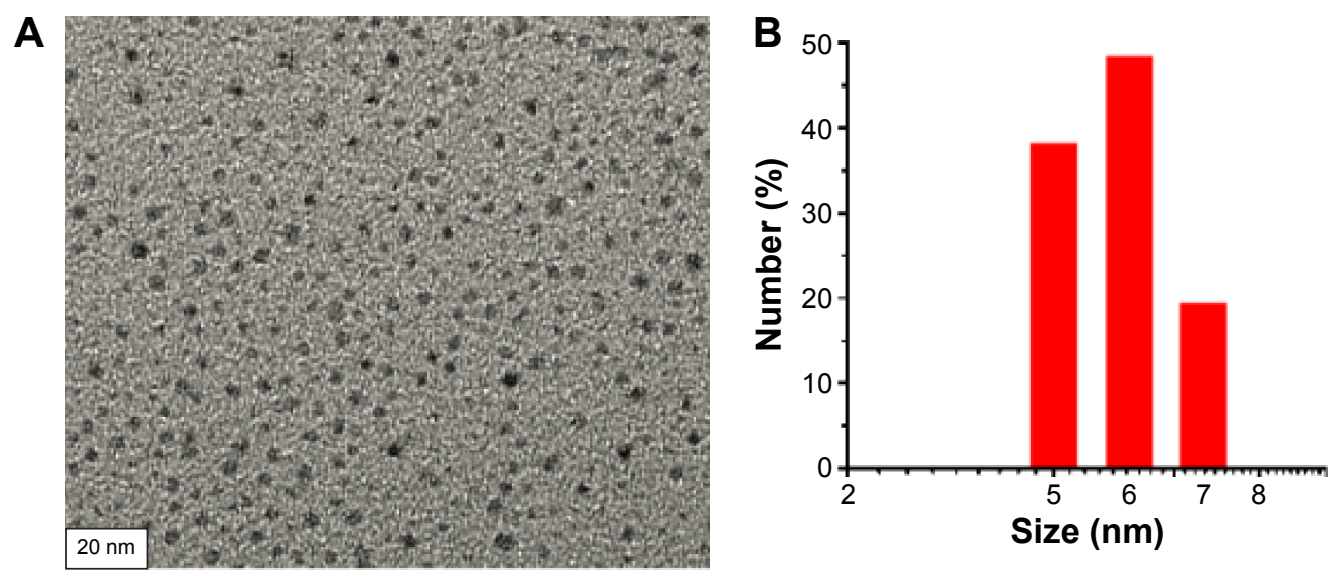

Figure 2 The shape and size of Gd-CDs.

Notes: (A) TEM image of Gd-CDs. The scale bar is $20 \mathrm{~nm}$. (B) Size distribution by the number of Gd-CDs.

Abbreviations: Gd-CDs, gadolinium-carbon dots; TEM, transmission electron microscope.

The chemical composition of Gd-CDs was additionally investigated by XPS. As shown in Figure 3A, the survey scan spectrum of as-prepared Gd-CDs exhibited the typical binding energies at the characteristic peaks of $\mathrm{C} 1 \mathrm{~s}, \mathrm{O} 1 \mathrm{~s}$, N1s, and Gd 3d, which confirmed the presence of carbon, oxygen, Gd, and nitrogen in the Gd-CDs. The characteristic
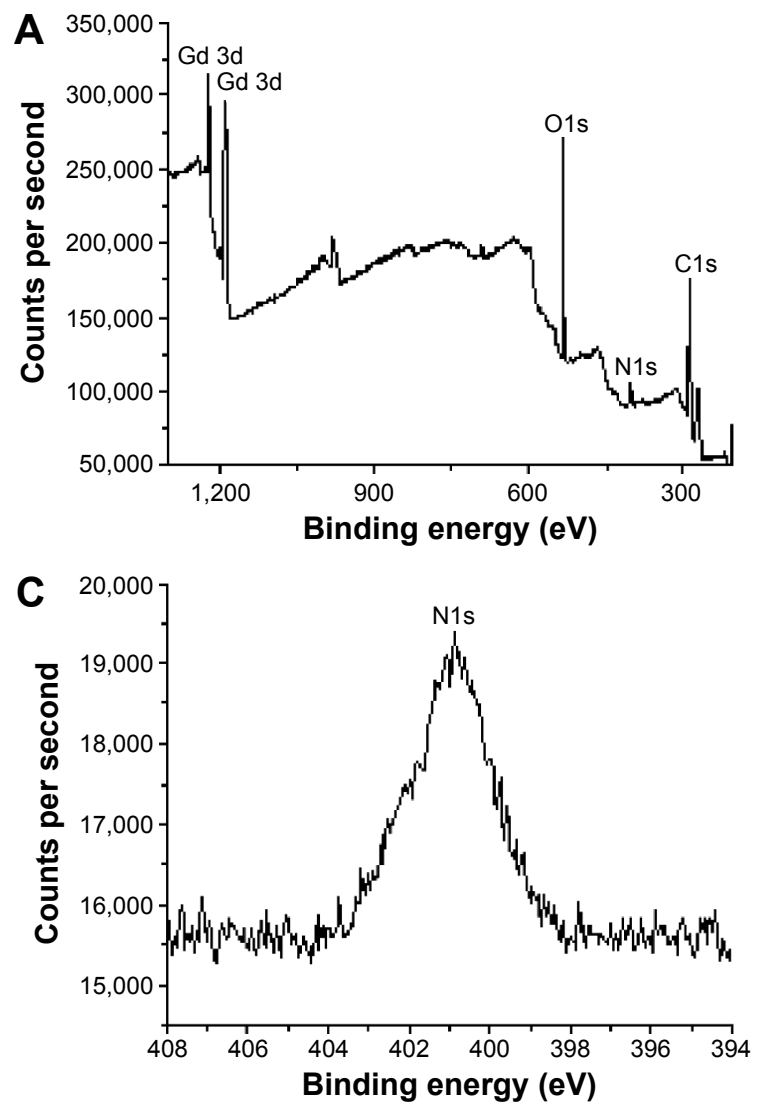

peaks corresponding to $\mathrm{C} 1 \mathrm{~s}(285.837 \mathrm{eV}$; Figure $3 \mathrm{~B})$ and N1s (401.98 eV; Figure 3C) were observed in the XPS survey spectrum. Meanwhile, the peaks for $\mathrm{Gd} 3 \mathrm{~d} 5 / 2$ and $\mathrm{Gd} 3 \mathrm{~d} 3 / 2$ were found at 1,187.425 eV and 1,220.105 eV (Figure 3D), respectively. Therefore, those peaks of standard $\mathrm{Gd}_{2} \mathrm{O}_{3}$ were reported at $1,188 \mathrm{eV}$ and $1,220 \mathrm{eV} \cdot{ }^{27}$ Such a subtle shift of the
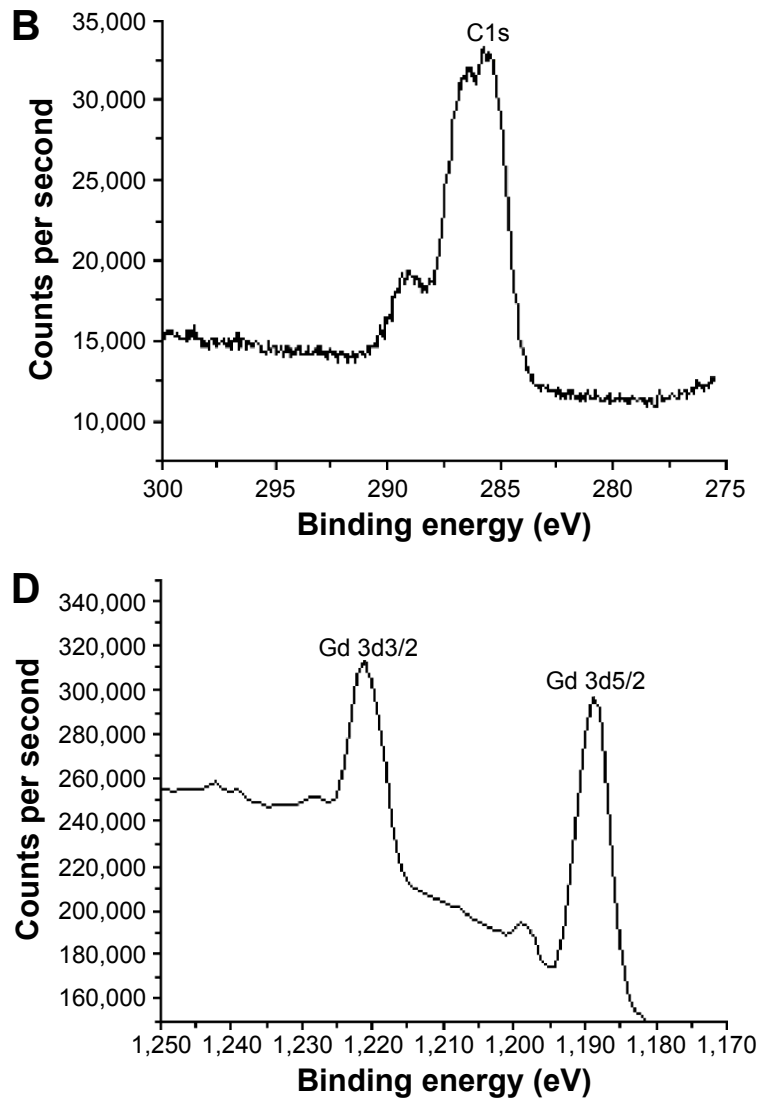

Figure 3 XPS spectrum of Gd-CDs.

Notes: (A) XPS survey spectrum; (B) XPS CIs spectrum; (C) XPS NIs spectrum; (D) XPS Gd 3d spectrum of Gd-CDs.

Abbreviations: Gd-CDs, gadolinium-carbon dots; XPS, X-ray photoelectron spectroscopy. 
binding energy is probably due to the different coordination environments. The changes in the channel of Gd validated the involvement of $\mathrm{Gd}$ ion into the CDs.

\section{Optical properties of Gd-CDs}

The as-prepared Gd-CDs under UV irradiation exhibited distinct blue luminescence and excellent aqueous dispersibility, which indicated that the CDs have good optical properties. The UV-visible absorption measurement showed a characteristic absorption peak at $360 \mathrm{~nm}$, which was attributed to the $\mathrm{n}-\pi^{*}$ transition of the $\mathrm{C}-\mathrm{N}$ band and the $\pi-\pi^{*}$ transition of the conjugated $\mathrm{C}=\mathrm{C}$ band. Furthermore, the emission wavelengths of Gd-CDs PL spectra display a progressive increase with increasing excitation wavelengths from $300 \mathrm{~nm}$ to $400 \mathrm{~nm}$ (Figure 4A). With the continued increase in the excitation wavelengths from $430 \mathrm{~nm}$ to $510 \mathrm{~nm}$, there appeared a relative steady variation of emission peaks, and the maximum emission peak at $485 \mathrm{~nm}$ was derived from the excitation wavelengths of $430 \mathrm{~nm}$ (Figure 4B). Gd-CDs showed a clear green fluorescence emission and unique fluorescence properties of CDs. Luminescence stability test showed that Gd-CDs were better than the QDs after placing under the light-source continuous irradiation for a period of time.

Significantly, the obtained Gd-CDs exhibited clear upconversion luminescence (UCL) properties, as shown in Figure 4C. When they were excited with long-wavelength light (from $760 \mathrm{~nm}$ to $860 \mathrm{~nm}$ ), the PL spectra showed a fixed emission peak at $475 \mathrm{~nm}$ that hardly shifted even when the excitation wavelength varied, a finding that was different from earlier reports. This phenomenon was attributed to absorbing two or more photons. ${ }^{28}$ Currently, there are two popular mechanisms accounting for the UCL of CDs, that is, the multiphoton active process and anti-Stokes PL.
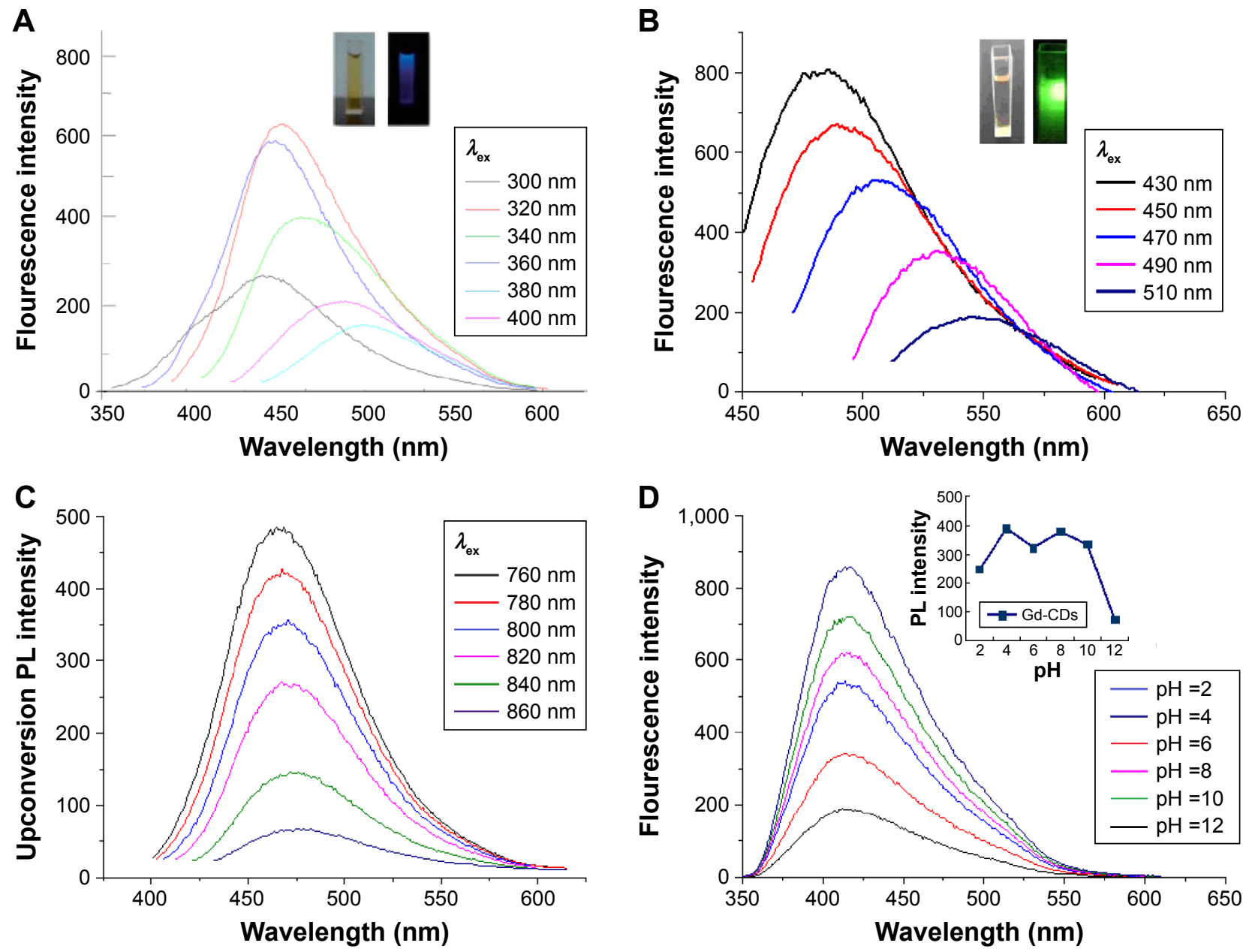

Figure 4 Fluorescence emission spectra of Gd-CDs with excitation wavelengths (A) from $300 \mathrm{~nm}$ to $400 \mathrm{~nm}$; (B) from $430 \mathrm{~nm}$ to $510 \mathrm{~nm}$; (C) from $760 \mathrm{~nm}$ to $860 \mathrm{~nm}$; (D) PL spectra of Gd-CDs at different $\mathrm{pH}$ values.

Notes: (A) Inset shows the color of Gd-CDs solution under sunlight and UV excitation light ( $365 \mathrm{~nm}$ ). (B) Inset shows the color of Gd-CDs solution under sunlight and visible excitation light $(450 \mathrm{~nm})$. (D) Inset: PL intensity as a function of $\mathrm{pH}$ PL spectra for Gd-CDs at $360 \mathrm{~nm}$ excitation for different $\mathrm{pH}$ values (the inset shows a good linearity in the $\mathrm{pH}$ range of 2.0-12.0).

Abbreviations: Gd-CDs, gadolinium-carbon dots; PL, photoluminescence. 
As the energy difference between the excitation and the UCL emission varies, the multiphoton active process seems more applicable for accounting for the UCL of Gd-CDs. These results indicate that CDs are expected to open new opportunities for cell and tissue imaging with two-photon luminescence microscopy. To further optimize the performance of Gd-CDs, we also examined the effect of $\mathrm{pH}$ on the fluorescence intensity of Gd-CDs. When the $\mathrm{pH}$ value was increased (Figure 4D), the Gd-CDs exhibited gradually strong PL emission. However, the luminescence intensity of Gd-CDs reached the strongest fluorescence emission at $\mathrm{pH}$ 4.0. Thus, the optimal $\mathrm{pH} 4.0$ may be useful for labeling tumor cells in a slightly acidic environment.

\section{Characterization of Gd-CDs/AFn (DOX)/FA}

AFn was derived from horse spleen ferritin after removing its internal soluble iron core. The inside diameter of the obtained AFn was $\sim 8 \mathrm{~nm}$ and the average outer diameter was $12 \mathrm{~nm}$. The combination of AFn-loaded DOX with Gd-CDs was achieved via amide bond formation. The surface of Gd-CDs was modified with amino groups, which was confirmed by FTIR and zeta potential detection. The zeta potential of Gd-CDs was $9.05 \mathrm{mV}$ (Figure 5A); the UV wavelength scanning results of Gd-CDs and Gd-CDs/AFn are shown in Figure 5B. A strong absorption peak at $355 \mathrm{~nm}$ from UV-visible absorption spectrum was observed, which was ascribed to the blue-shifted $\pi-\pi^{*}$ transition of the amino groups on the surface of Gd-CDs. After the attachment of Gd-CDs to the surface of AFn, UV-visible spectrum showed two peaks at $355 \mathrm{~nm}$ and $268 \mathrm{~nm}$, which was probably due to the interactions between the functional groups of AFn and the amino groups of the Gd-CDs, resulting in the blue shift of AFn in the UV-visible region. Pure DOX had an obvious peak at $480 \mathrm{~nm}$ in the UV absorption spectrum. Similarly, DOX loaded with AFn showed two peaks at $280 \mathrm{~nm}$ and

A

\begin{tabular}{|c|c|c|c|}
\hline Parameters & Mean $(\mathrm{mV})$ & Area (\%) & St dev $(\mathrm{mV})$ \\
\hline Zeta potential $(\mathrm{mV}): \quad 9.05$ & Peak 1:9.05 & 100 & 3.67 \\
\hline Zeta deviation $(\mathrm{mV}): \quad 3.67$ & Peak 2:0.00 & 0.0 & 0.00 \\
\hline Conductivity (mS/cm): 0.0425 & Peak 3:0.00 & 0.0 & 0.00 \\
\hline Result quality & & & \\
\hline
\end{tabular}
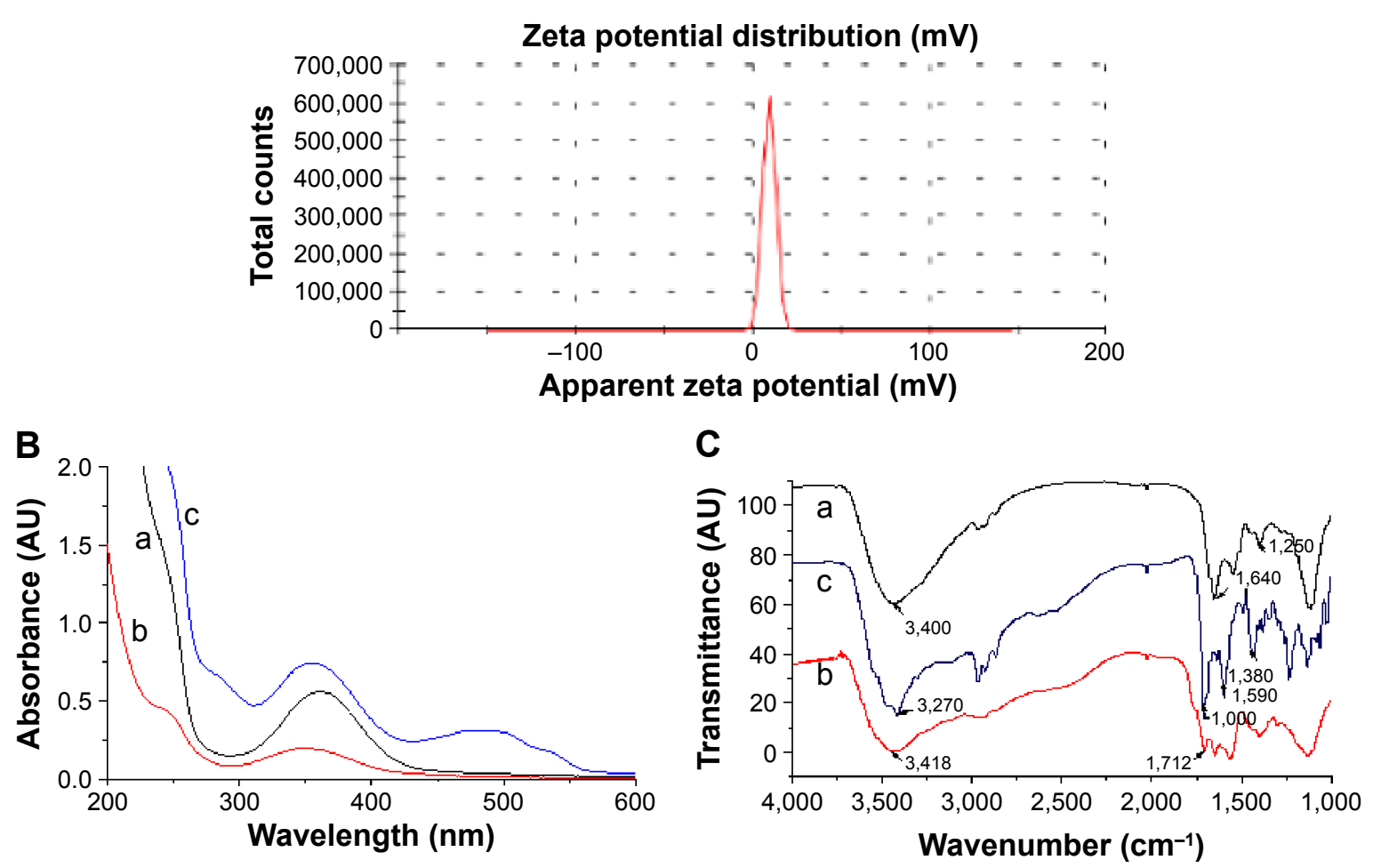

Figure 5 Characterization of Gd-CDs/AFn(DOX)/FA.

Notes: (A) Zeta potential values of Gd-CDs; (B) The Gd-CDs/AFn (DOX) complex UV-visible spectra of Gd-CDs and its complexes after surface modification: (a) Gd-CDs, (b) Gd-CDs/AFn, (c) DOX-loaded Gd-CDs/AFn complex; (C) FTIR spectrum of (a) Gd-CDs, (b) AFn, (c) Gd-CDs/AFn.

Abbreviations: AFn, apoferritin; DOX, doxorubicin; FTIR, Fourier transform infrared; Gd-CDs, gadolinium-carbon dots; St dev, standard deviation; UV, ultraviolet. 
$480 \mathrm{~nm}$, which indicated that DOX was successfully encapsulated in the AFn.

The FTIR spectra of Gd-CDs (Figure 5C) revealed the presence of multiple surface-passivated groups. Some of the prominent bands were as follows: the band at $1,640 \mathrm{~cm}^{-1}$ brought about the stretching mode of $-\mathrm{NH}$ and another band at $1,250 \mathrm{~cm}^{-1}$ was assigned to the symmetric stretching mode of $-\mathrm{CN}$. The abovementioned bands confirmed the presence of numerous amino groups at the surface of CDs. The FTIR spectra of AFn showed three characteristic peaks located in hydroxyl at $3,400 \mathrm{~cm}^{-1}$; carbonyl at $1,712 \mathrm{~cm}^{-1}$; and amino at $1,650 \mathrm{~cm}^{-1},{ }^{29}$ which were the skeleton characteristic peaks of AFn nanocages. After attaching Gd-CDs, some prominent bands confirming the interaction between Gd-CDs and AFn were as follows: the infrared bands of Gd-CDs/AFn complex appeared as amino bond (I) at $1,660 \mathrm{~cm}^{-1}$ and amino bond (II) at $1,590 \mathrm{~cm}^{-1}, 30$ which were due to an aromatic
$\mathrm{C}=\mathrm{O}$ stretching vibration peaks, shear vibration amide $\mathrm{N}-\mathrm{H}$ bend, and an anhydride $\mathrm{C}=\mathrm{O}$ stretch between the amino acids and amino group at the surface of Gd-CDs. The bands at $1,380 \mathrm{~cm}^{-1}(-\mathrm{NH})$ came from bending vibration peak of amino acids. ${ }^{31,32}$ All the abovementioned peaks confirmed that Gd-CDs were successfully attached to the surface of AFn through the amidation process.

We found that Gd-CDs/AFn (DOX)/FA tend to form monodisperse aggregates in the size range of 100-200 nm as confirmed by DLS and TEM. The size and zeta potential distribution of Gd-CDs/AFn (DOX)/FA are shown in Figure 6. The average particle size of Gd-CDs/AFn (DOX)/ FA was $112 \pm 3.5 \mathrm{~nm}$ (Figure 6A), and the average potential was $-23.7 \pm 2.1 \mathrm{mV}$ (Figure 6B). The morphology of Gd-CDs/ AFn (DOX)/FA was characterized by TEM. It was observed that Gd-CDs/AFn (DOX)/FA have a ball-like structure (Figure 6C) and the size was $\sim 100 \mathrm{~nm}$, and AFn nanocage
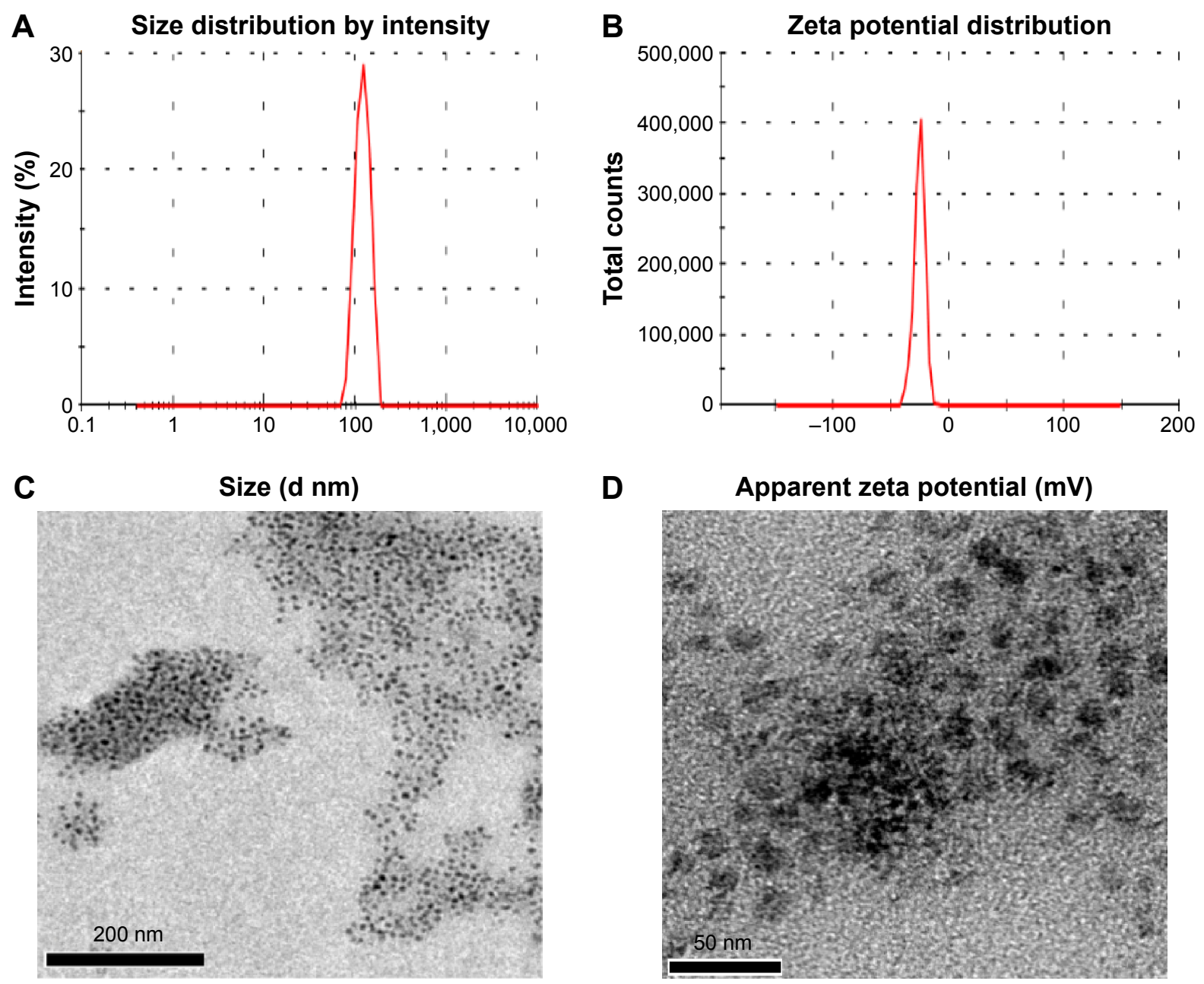

Figure 6 The size, zeta potential, and TEM images of nanoparticles.

Notes: (A) and (B) DLS analysis results of Gd-CDs/AFn (DOX)/FA. (C) and (D) TEM image of Gd-CDs/AFn (DOX)/FA.

Abbreviations: AFn, apoferritin; DLS, dynamic light scattering; DOX, doxorubicin; FA, folic acid; Gd-CDs, gadolinium-carbon dots; TEM, transmission electron microscope. 
was covered with a large number of black carbon QDs. TEM images (Figure 6D) indicated that the nanoparticles have a uniform size and a ball-like structure, which confirmed that Gd-CDs/AFn (DOX)/FA have good water solubility and can be stably dispersed in water, cell culture fluid, and in vivo.

\section{In vitro MRI}

The nanocomposites can act as a $\mathrm{T}_{1}$ contrast agent for MRI owing to the presence of $\mathrm{Gd}^{3+}$ inside of Gd-CDs/AFn (DOX)/FA. Gd has been widely used as a $\mathrm{T}_{1}$ contrast agent in MRI. ${ }^{33,34} \mathrm{~T}_{1}$-weighted MR images of Gd-CDs/AFn (DOX)/ FA solutions acquired on a $3 \mathrm{~T}$ MR scanner revealed the concentration-dependent whitening effect. It can be easily seen from Figure 7A that the brightness became more prominent upon increasing the concentration of Gd-CDs/AFn (DOX)/FA. As plotted in Figure 7B, both $R_{1}$ and $R_{2}$ values of Gd-CDs/AFn (DOX)/FA were enhanced linearly with the equivalently increased Gd concentration. Accordingly, the $R_{1}$ and $R_{2}$ relaxation rates of Gd-CDs/AFn (DOX)/FA were calculated to be $11.429 \mathrm{~mL} \mathrm{mg}^{-1} \mathrm{~s}^{-1}$ and $15.328 \mathrm{~mL} \mathrm{mg}^{-1} \mathrm{~s}^{-1}$, respectively. Moreover, the $R_{2} / R_{1}$ ratio of 1.35 further suggested that Gd-CDs/AFn (DOX)/FA is an effective $T_{1}$ contrast agent.

\section{Drug release kinetics}

The Gd-CDs/AFn (DOX)/FA compound showed an ideal drug release profile at physiological as well as slightly acidic
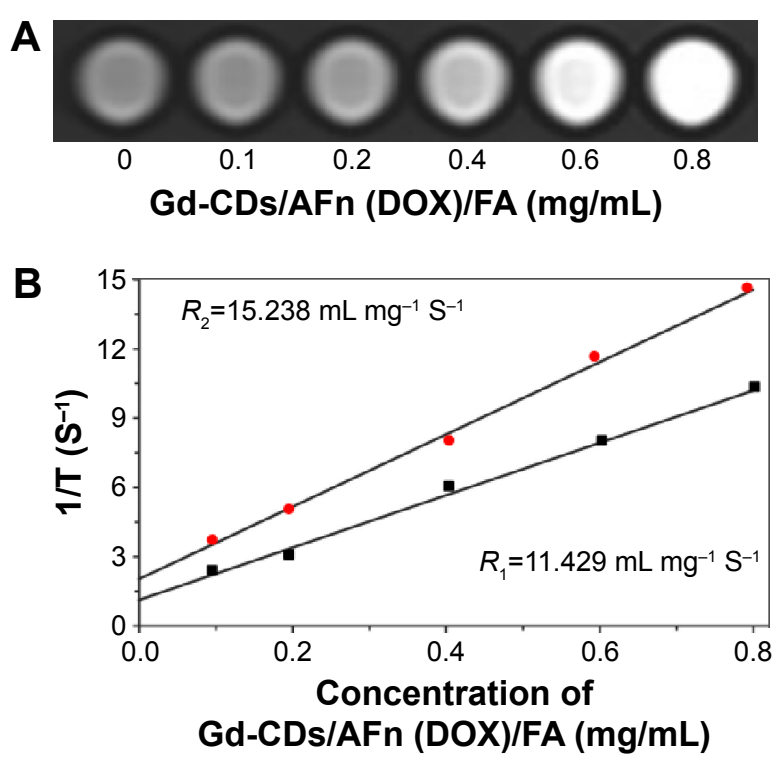

Figure 7 Magnetic properties of Gd-CDs/AFn (DOX)/FA.

Notes: (A) $T_{1}$-weighted MR images of Gd-CDs/AFn (DOX)/FA solutions at different concentrations; (B) linear relationship $R$ of Gd-CDs/AFn (DOX)/FA solutions at different concentrations.

Abbreviations: AFn, apoferritin; DOX, doxorubicin; FA, folic acid; Gd-CDs, gadolinium-carbon dots; $\mathrm{MR}$, magnetic resonance; $R$, relaxation rate.
$\mathrm{pH}$ conditions and was found to follow first-order release kinetics based on the statistical calculations. The drug release curves of Gd-CDs/AFn (DOX)/FA in PBS ( $\mathrm{pH}=7.4)$ and acetate buffer $(\mathrm{pH}=5.0)$ are shown in Figure 8A. The results showed that the drug release of DOX was more favorable under the condition of $\mathrm{pH} 5.0$. At $\sim 72$ hours, the cumulative release rate was $79.2 \%$. However, there was a slower release under the condition of $\mathrm{pH} 7.4$; the final cumulative release rate was only $18.1 \%$ at 72 hours, which indicated that DOX release from Gd-CDs/AFn (DOX)/FA has high $\mathrm{pH}$ sensitivity. The results were considered to be consistent with another use of hydrazone bond to construct $\mathrm{pH}$-sensitive drug delivery system. ${ }^{35}$ DOX almost cannot be released from AFn nanocages in normal tissues due to the $\mathrm{pH} 7.4$ of bodily fluid environment. As for partial acid environment of the tumor tissue, a large number of DOX molecules were slowly released from AFn nanocages after Gd-CDs/AFn (DOX)/FA worked on the tumor position, which greatly improved the treatment efficiency of DOX.

\section{Cell cytotoxicity}

The cytotoxicity study of Gd-CDs and Gd-CDs/AFn on MCF-7 cells is shown in Figure 8B. The concentration of Gd-CDs was within the range of 6-200 $\mu \mathrm{g} / \mathrm{mL}$. The cytotoxicity testing revealed that the survival rate of MCF-7 cells was still $>85 \%$. In the following experiment, the concentration of Gd-CDs was generally $<10 \mu \mathrm{g} / \mathrm{mL}$, and the survival rate of $\mathrm{MCF}-7$ cells was $\sim 95 \%$. Thus, Gd-CDs had no effect on the growth of MCF-7 cells, and there was no obvious cytotoxicity. The obtained Gd-CDs not only increased the quantum yield but also greatly decreased the toxicity of $\mathrm{Gd}^{3+}$ in vivo under the protection of carbon layer structure. After Gd-CDs attached to AFn, there appeared a slight increase in biocompatibility compared with Gd-CDs alone, which showed that blank carrier of Gd-CDs/AFn was highly biocompatible.

\section{Cellular uptake}

To investigate the ability of FA targeting MCF-7 cells after modification of nanoparticles with FA, MCF-7 cells were incubated with Gd-CDs, Gd-CDs/AFn (DOX), and Gd-CDs/ AFn (DOX)/FA for 1 hour, 2 hours, or 4 hours in cell culture medium. Figure 9 shows the results of confocal images acquired on isolated MCF-7 cells. There was no significant difference among all time points of the Gd-CDs group, suggesting that Gd-CDs alone could not enter into cancer cells at the set time points because of positive charge on the surface of CDs, but they just gathered on the cell surface. 

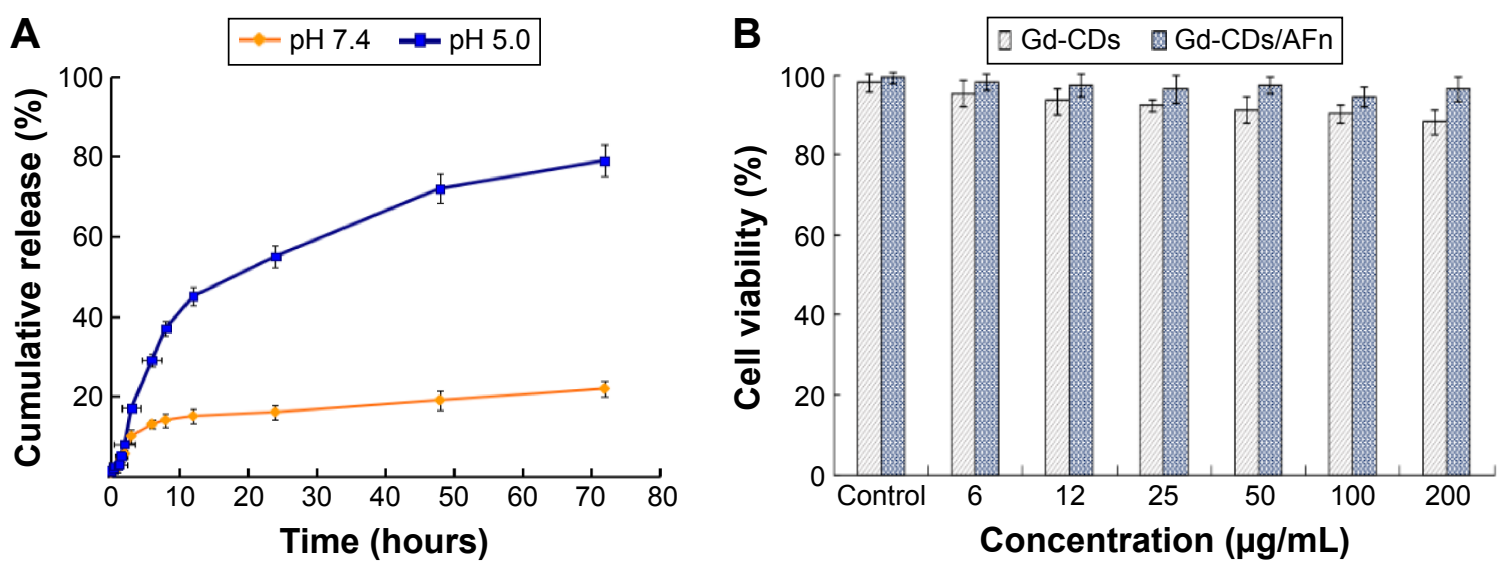

Figure 8 The drug release curves and cytotoxicity study.

Notes: $(\mathbf{A})$ In vitro cumulative release of $\mathrm{Gd}-\mathrm{CD} / \mathrm{AFn}(\mathrm{DOX}) / \mathrm{FA}$ results with respect to time. Data are presented as mean \pm standard deviation ( $\mathrm{n}=3$ ). (B) Effects of Gd-CDs and Gd-CDs/AFn with varied concentrations on the viability of MCF-7 cells.

Abbreviations: AFn, apoferritin; DOX, doxorubicin; FA, folic acid; Gd-CDs, gadolinium-carbon dots.

Green fluorescence was detected in cells treated with both Gd-CDs/AFn (DOX) and Gd-CDs/AFn (DOX)/FA, which showed that both Gd-CDs/AFn (DOX) and Gd-CDs/AFn $(\mathrm{DOX}) / \mathrm{FA}$ can enter into MCF-7 cells through the cell membrane. However, MCF-7 cells have a significant time dependence on the uptake of nanoparticles. As for Gd-CDs/ AFn (DOX), the nucleus appeared not to be affected by the drug at 1 hour, while only a minimal amount of drug could be detected inside the nucleus at 2 hours. In contrast, Gd-CDs/ AFn (DOX)/FA could efficiently deliver a detectable amount

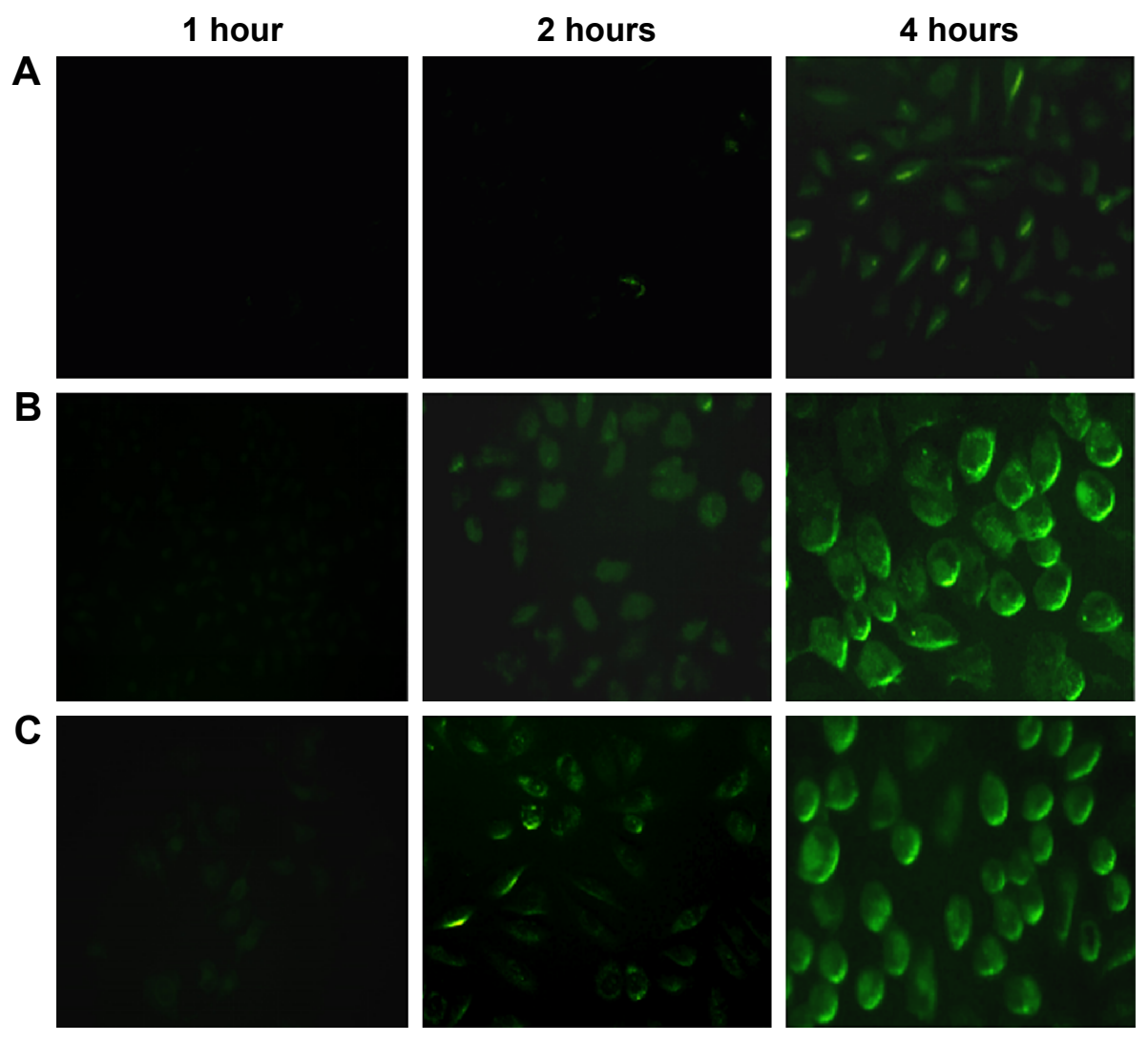

Figure 9 Doxorubicin release inside the nuclear compartment of MCF-7 cells.

Notes: Confocal microscopy images of MCF-7 cells incubated with I $\mu$ M of (A) Gd-CDs, (B) Gd-CDs/AFn (DOX), and (C), Gd-CDs/AFn (DOX)/FA for I hour, 2 hours, or 4 hours at $37^{\circ} \mathrm{C}$. Magnification: $200 \times$.

Abbreviations: AFn, apoferritin; DOX, doxorubicin; FA, folic acid; Gd-CDs, gadolinium-carbon dots. 
of drug into the nucleus quickly at 2 hours, and more green fluorescence signal of Gd-CDs could be observed at 4 hours with the extension of the incubation time. The results indicated that Gd-CDs/AFn (DOX)/FA could effectively promote cellular uptake, and this distinction in uptake was probably due to the presence of FA.

\section{Inhibition efficiency on MCF-7 cells}

MCF-7 cells were treated with Gd-CDs/AFn (DOX)/FA, and their viability was evaluated in the presence of $20 \mu \mathrm{L}$ MTT. The kind of inhibitor and its working concentration have been selected to obtain the maximal percentage of multidrug resistance inhibition without affecting cell viability. The results are shown in Figure 10, which indicated that the proliferation and inhibition properties of Gd-CDs/AFn (DOX), Gd-CDs/AFn (DOX)/FA, and free DOX were evident on MCF-7 cells. Both the preparation groups and the bare DOX groups exhibited inhibition of proliferation of MCF-7 cells, and the inhibition rate was significantly dependent on the dose of DOX and cultivation time. After cultivation for 24 hours, when the concentration of DOX was $10 \mu \mathrm{g} / \mathrm{mL}$, the inhibition rate of Gd-CDs/AFn (DOX)/FA on MCF-7 cells was higher than that of free DOX and Gd-CDs/ AFn (DOX). But when the incubation time was 48 hours or 72 hours, the inhibition rate of both Gd-CDs/AFn (DOX)/FA and Gd-CDs/AFn (DOX) on MCF-7 cells was higher than that of free DOX. Gd-CDs/AFn (DOX)/FA could deliver more DOX into MCF-7 cells with the help of tumor targeting, which indicated that Gd-CDs/AFn (DOX)/FA can enhance the inhibition of MCF-7 cell proliferation.

\section{In vivo cancer therapy}

The relative tumor volume change curves of tumor-bearing mice in each group during 14 days of treatment are shown in Figure 11A. The groups of Gd-CDs/AFn (DOX) and Gd-CDs/AFn (DOX)/FA could gradually inhibit tumor from growing compared with the same dose of clinically approved DOX, especially in the group of FA-mediated targeting. It could be seen that the inhibition ability of Gd-CDs/AFn $(\mathrm{DOX}) / \mathrm{FA}$ on tumor was obviously better than that of free DOX $(P<0.05)$, which showed that the nano-drug delivery system can make more drugs to reach the tumor site, through the active targeting of FA, resulting in a better antitumor effect. In contrast, the physiological saline and Gd-CDs/ AFn groups have nearly no inhibition on tumor growth. Their average tumor volume rapidly reached $10.2 \pm 1.2 \mathrm{~mm}^{3}$ at 14 days after tumor implantation. As already known, the increase in drug toxicity in vivo usually leads to the loss of body weight, and hence the weight of mice in each group was examined. During the process of treatment with the drug for
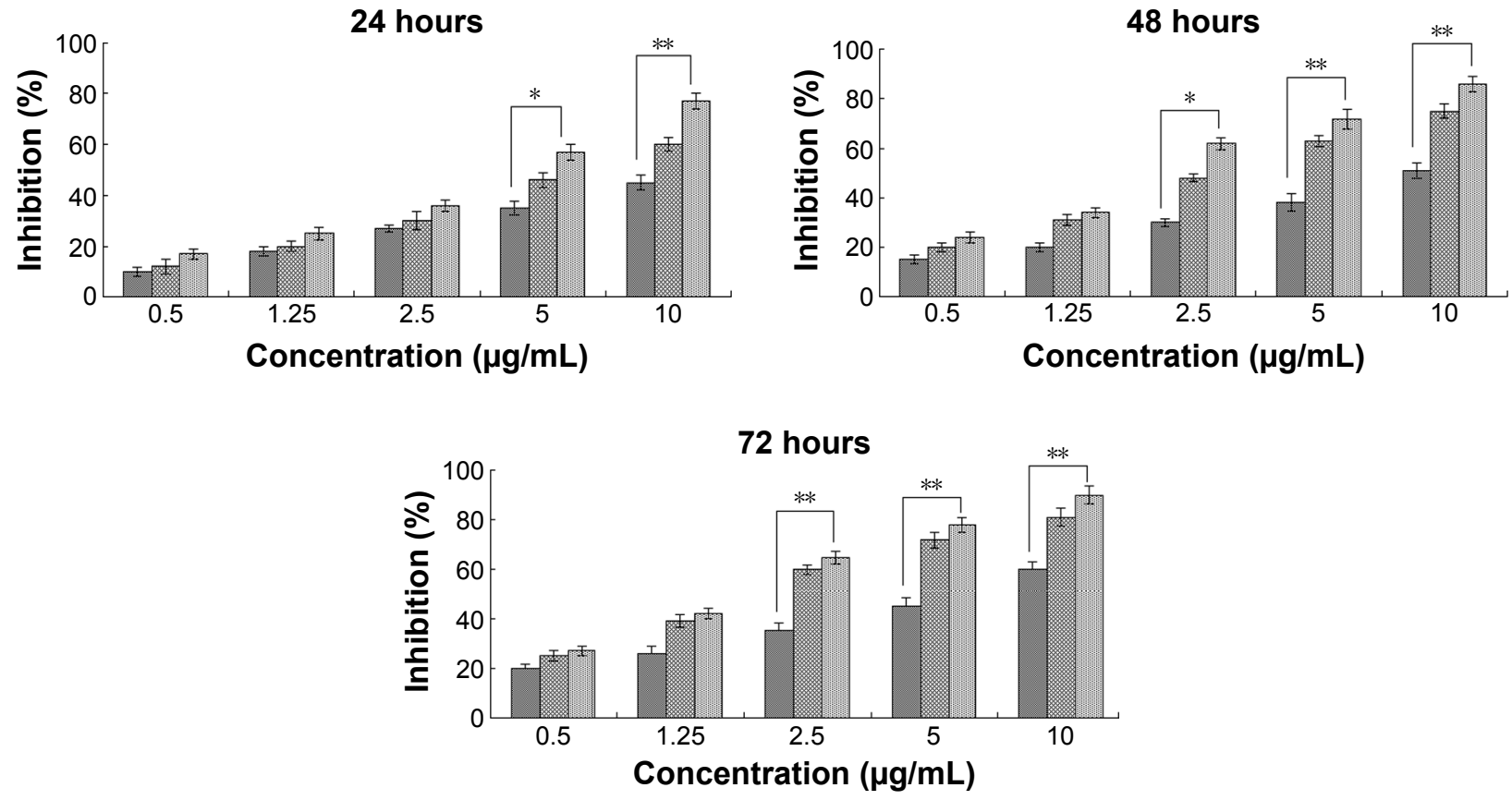

DOX Gd-CDs/AFn (DOX) 国 Gd-CDs/AFn (DOX)/FA

Figure 10 Inhibition efficiency of DOX, Gd-CDs/AFn (DOX), and Gd-CDs/AFn (DOX)/FA on MCF-7 cells.

Notes: Data are presented as mean \pm standard deviation $(n=3)$. $* P<0.05 ; * * P<0.01$.

Abbreviations: AFn, apoferritin; DOX, doxorubicin; FA, folic acid; Gd-CDs, gadolinium-carbon dots. 
A

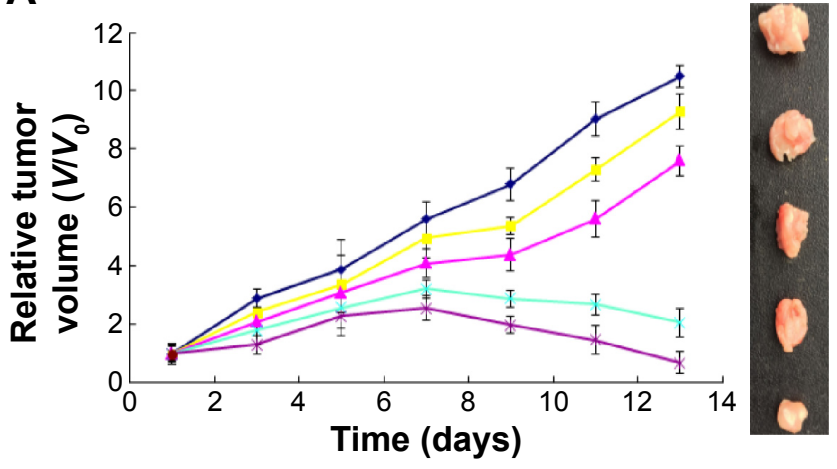

B

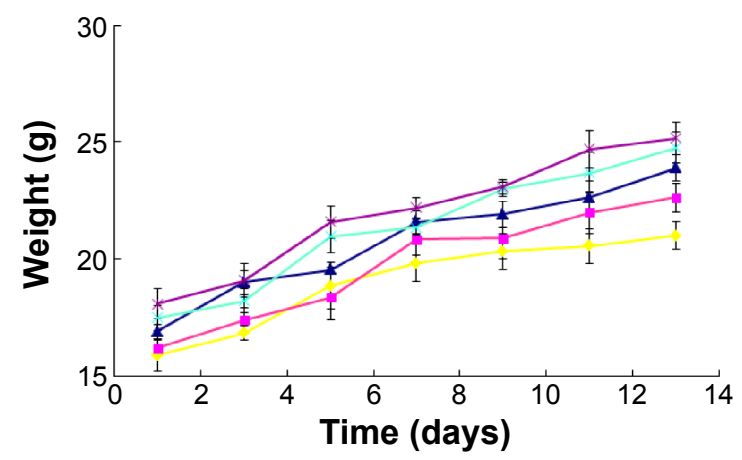
$\rightarrow$ Saline $\rightarrow$ Gd-CDs/AFn $\rightarrow$ DOX - Gd-CDs/AFn (DOX) $\rightarrow$ Gd-CDs/AFn (DOX)/FA

C

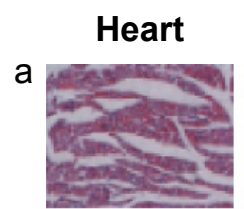

Liver
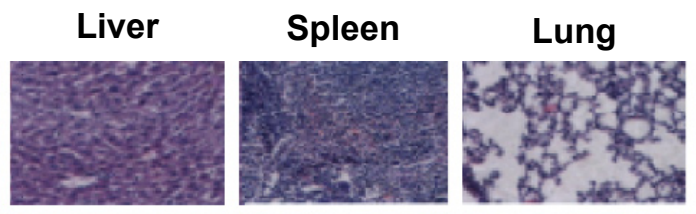

Kidney

Tumor

b
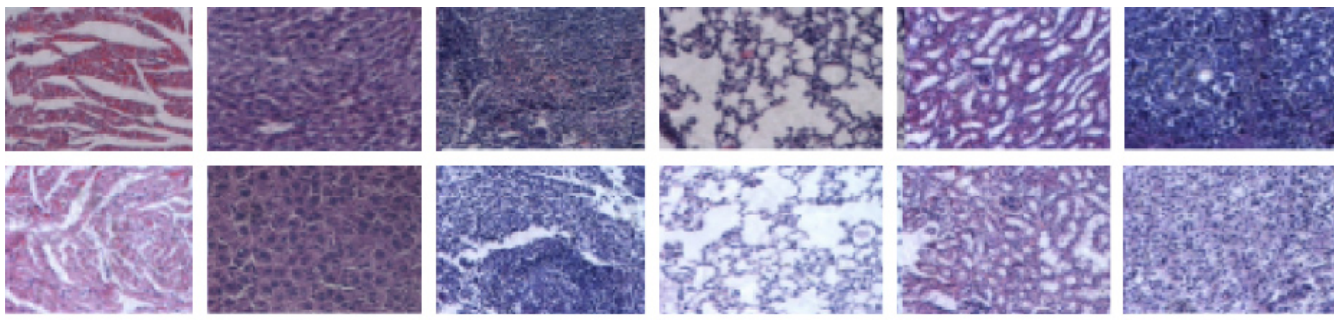

C
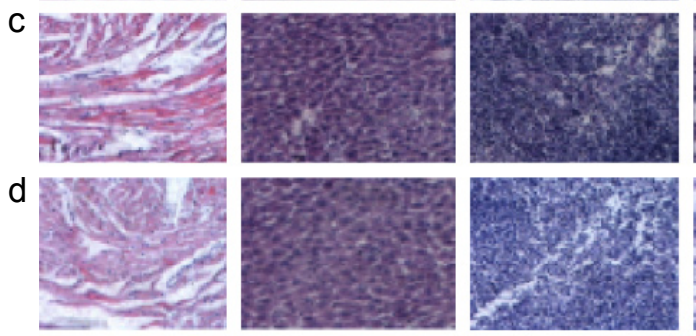

e
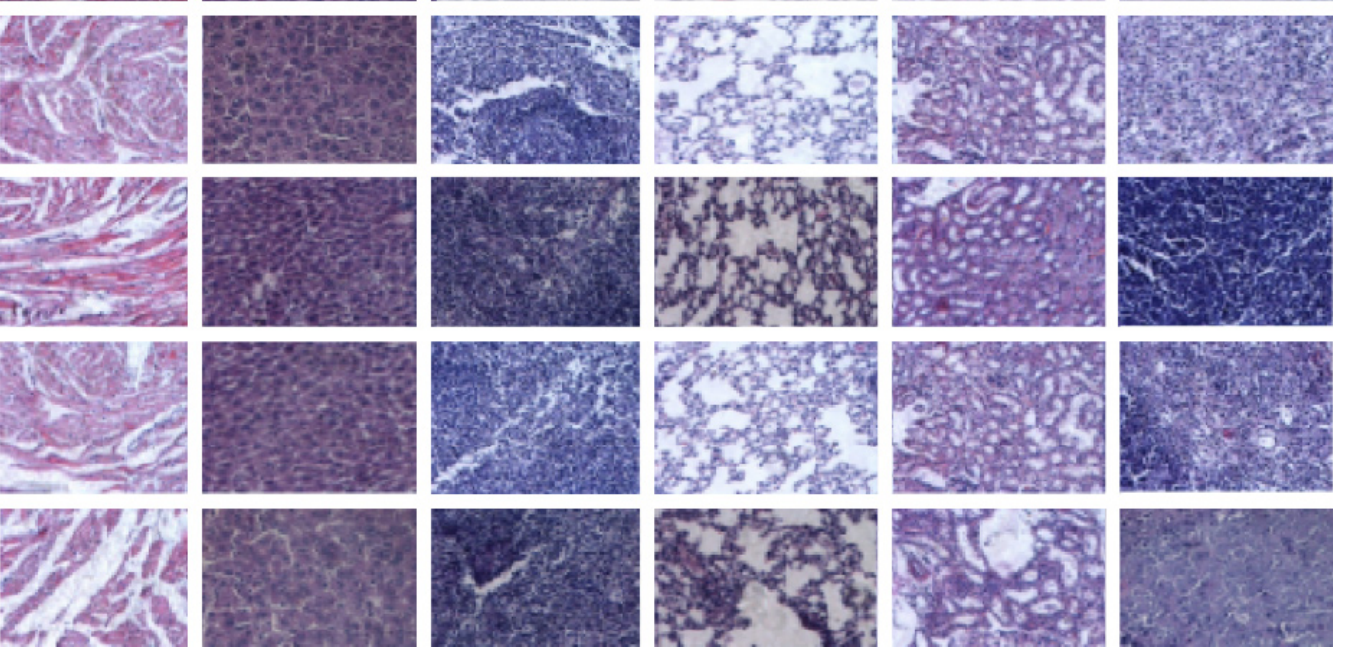

Figure I I In vivo treatments.

Notes: (A) Tumor growth of mice in different treatment groups within 14 days ( $V_{0}$, initial tumor volume, $\left.P<0.05\right)$. (B) Changes in body weight of mice in different groups during treatment. (C) H\&E-stained tumor tissues harvested from the mice with different treatments. (a) Control, (b) Gd-CDs/AFn, (c) DOX, (d) Gd-CDs/AFn (DOX), and (e) Gd-CDs/AFn (DOX)/FA. Magnification: 200x. Data are presented as mean \pm standard deviation $(n=6)$.

Abbreviations: AFn, apoferritin; DOX, doxorubicin; FA, folic acid; Gd-CDs, gadolinium-carbon dots; H\&E, hematoxylin and eosin.

14 days, the weight of all mice was measured and recorded, and then the variations in weight during the treatment process were plotted as a curve, as shown in Figure 11B. The results showed that the weight of mice was not affected by the five treatment groups, which also indicated that almost no toxicity was observed in all the treatment groups.

To further study the toxicity of the nanocomposites on the important organs of tumor-bearing mice, the organs of mice in each group were examined using hematoxylinand-eosin-stained pathological sections. The cell structure of each tissue was observed under the optical microscope, and the results are shown in Figure 11C. It can be observed that the drug-delivered groups exhibited varying degrees of damage on the tumor tissue and cells compared with the saline group. Different degrees of reduction and apoptosis of tumor cells were observed by microscope, especially in the FA-targeted therapy group. However, the group of no FA targeting was less damage than the group with FA targeted therapy. These results further validated the enhancement of therapeutic effect in the Gd-CDs/AFn (DOX)/FA group through FA, which accorded with the results and its effect on tumor volume and weight.

\section{Pharmacokinetics}

To investigate the pharmacokinetics of various drug complexes, blood samples of C57 mice after injection of the 


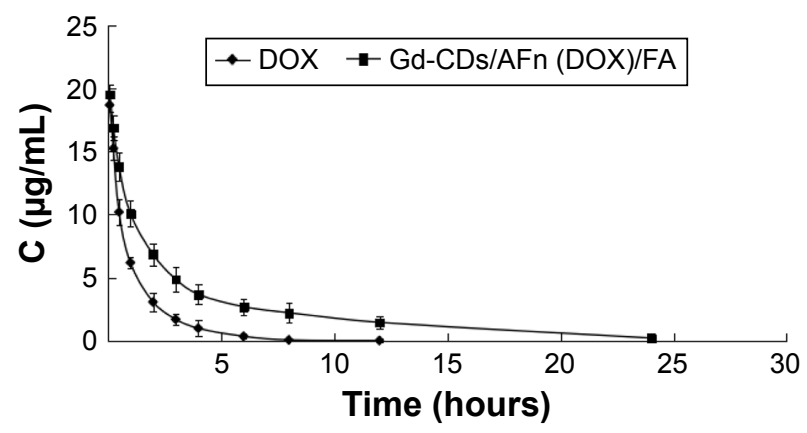

Figure 12 The results of drug-time curve.

Note: Mean concentration of DOX in plasma after intravenous administration of DOX and Gd-CDs/AFn (DOX)/FA.

Abbreviations: AFn, apoferritin; DOX, doxorubicin; FA, folic acid; Gd-CDs, gadolinium-carbon dots.

Gd-CDs/AFn (DOX)/FA or free DOX at different time points were assessed by high-performance liquid chromatography. The pharmacokinetic parameters were calculated by the two-compartment model. There were significant differences in the pharmacokinetics of the drug in vivo. As shown in the drug-time curve (Figure 12), the decrease in free DOX concentration was faster than that achieved with administration of Gd-CDs/AFn (DOX)/FA. The area under the curve (AUC) of the Gd-CDs/AFn (DOX)/FA $(58.91[\mu \mathrm{g} / \mathrm{mL}] \cdot$ hours $)$ was about two times higher than that of free DOX $(21.40[\mu \mathrm{g} / \mathrm{mL}]$.hours $)$. The results showed that Gd-CDs/AFn (DOX)/FA greatly increased the bioavailability of DOX in vivo. The elimination rates of free DOX and Gd-CDs/AFn (DOX)/FA were $0.23(\mathrm{mg} / \mathrm{kg}) /(\mu \mathrm{g} / \mathrm{mL})$-hours and $0.08(\mathrm{mg} / \mathrm{kg}) /(\mu \mathrm{g} / \mathrm{mL}) \cdot$ hours, respectively (Table 1$)$, which showed Gd-CDs/AFn (DOX)/FA had a significant release effect. The mean residence time of the Gd-CDs/AFn (DOX)/FA (5.32 hours) was two times longer than that of free DOX (1.52 hours), indicating that Gd-CDs/AFn (DOX)/ FA greatly prolonged the half-life and blood circulation time of DOX in vivo.

\section{In vivo $M R I$}

To investigate the feasibility of nanocomposites as a $T_{1}$ contrast agent for MRI, S180 tumor-bearing mice were examined at 1 hour, 3 hours, and 6 hours after injection of Gd-CDs/AFn (DOX)/FA (Figure 13A). Saline group was used as a control. It was observed that the subcutaneous tumor appeared hyper-intense at 1 hour post injection. Subsequently, an obvious whitening effect on the tumor was observed in $\mathrm{T}_{1}$-weighted MR images for 3 hours. After injection of Gd-CDs/AFn (DOX)/FA for 6 hours, the signal intensities of tumor were reduced remarkably. In other words, after injecting with Gd-CDs/AFn (DOX)/FA for
Table I Pharmacokinetic parameters of DOX after intravenous administration of DOX and Gd-CDs/AFn (DOX)/FA in mice $(n=5)$

\begin{tabular}{llll}
\hline Parameters & Unit & DOX & $\begin{array}{l}\text { Gd-CDs/AFn } \\
\text { (DOX)/FA }\end{array}$ \\
\hline $\mathrm{T}_{1 / 2 \alpha}$ & hours & 0.26 & 0.49 \\
$\mathrm{~T}_{1 / 2 \beta}$ & hours & 1.33 & 3.79 \\
$\mathrm{AUC}$ & $(\mu \mathrm{g} / \mathrm{mL}) \cdot$ hours & 21.40 & 58.91 \\
$\mathrm{AUMC}$ & $(\mu \mathrm{g} / \mathrm{mL}) \cdot$ hours $^{2}$ & 32.61 & 333.96 \\
$\mathrm{~K}_{12}$ & hours $^{-1}$ & 0.59 & 0.50 \\
$\mathrm{~K}_{21}$ & hours $^{-1}$ & 1.61 & 0.50 \\
$\mathrm{~K}_{10}$ & hours $^{-1}$ & 0.97 & 0.38 \\
$\mathrm{MRT}$ & hours & 1.52 & 5.32 \\
$\mathrm{CL}$ & $(\mathrm{mg} / \mathrm{kg}) /(\mu \mathrm{g} / \mathrm{mL}) \cdot$ hours & 0.23 & 0.08 \\
$\mathrm{Vss}$ & $(\mathrm{mg} / \mathrm{kg}) /(\mu \mathrm{g} / \mathrm{mL})$ & 0.48 & 0.52
\end{tabular}

Note: $T_{1 / 2 \alpha}$ is the distribution half-life; $T_{1 / 2 \beta}$ is the elimination half-life; Vss is the apparent volume of the plasma compartment; and $\mathrm{K}_{10}, \mathrm{~K}_{12}$, and $\mathrm{K}_{21}$ are the apparent first order rate constant from the central division, the first order transfer rate constant from the central chamber to the outer chamber, and the first order transfer rate constant from the outer chamber to the central chamber, respectively. Abbreviations: AFn, apoferritin; AUC, area under the curve; AUMC, area under the first moment curve; DOX, doxorubicin; FA, folic acid; Gd-CDs, gadoliniumcarbon dots; MRT, mean residence time.

1 hour, 3 hours, and 6 hours, the MRI signal of the tumor increased by $32.5 \% \pm 3.2 \%, 71.6 \% \pm 4.5 \%$, and $15.3 \% \pm 3.6 \%$ compared with the control group, respectively (Figure 13B). Consequently, these results suggested that the Gd-CDs/ AFn $(\mathrm{DOX}) / \mathrm{FA}$ can act as a suitable negative $\left(\mathrm{T}_{1}\right)$ contrast agent in MRI applications.

\section{Conclusion}

Luminescent Gd-CDs were prepared as a probe for labeling AFn (DOX) nanoparticles with excellent fluorescence properties. Cell toxicity test results showed that Gd-CDs can be used as fluorescent probes with good biocompatibility and lower toxicity to biological cells and tissues. Besides, $\mathrm{Gd}$ ion $\left(\mathrm{Gd}^{3+}\right)$-doped $\mathrm{CDs}$ extend the potential application of Gd-CDs in MRI. AFn nanocages have the advantage of carrying high doses of DOX for tumor-specific targeting and killing tumor cells, with FA acting as a targeting ligand. Tumor inhibition experiments showed that Gd-CDs/AFn (DOX)/FA complex has a higher inhibition rate on cancer cells than that of free DOX and was found to be less toxic to normal cells due to FA-mediated targeting. Relative high loading capacity and good water solubility make AFn a promising carrier for anticancer drugs. In vitro and in vivo studies showed that the Gd-CDs/AFn (DOX)/FA has advantages such as excellent fluorescence, obvious magnetic property, good biocompatibility, and tumor targeting ability, which indicated the great potential of Gd-CDs/AFn (DOX)/FA for cancer theranostic applications. 

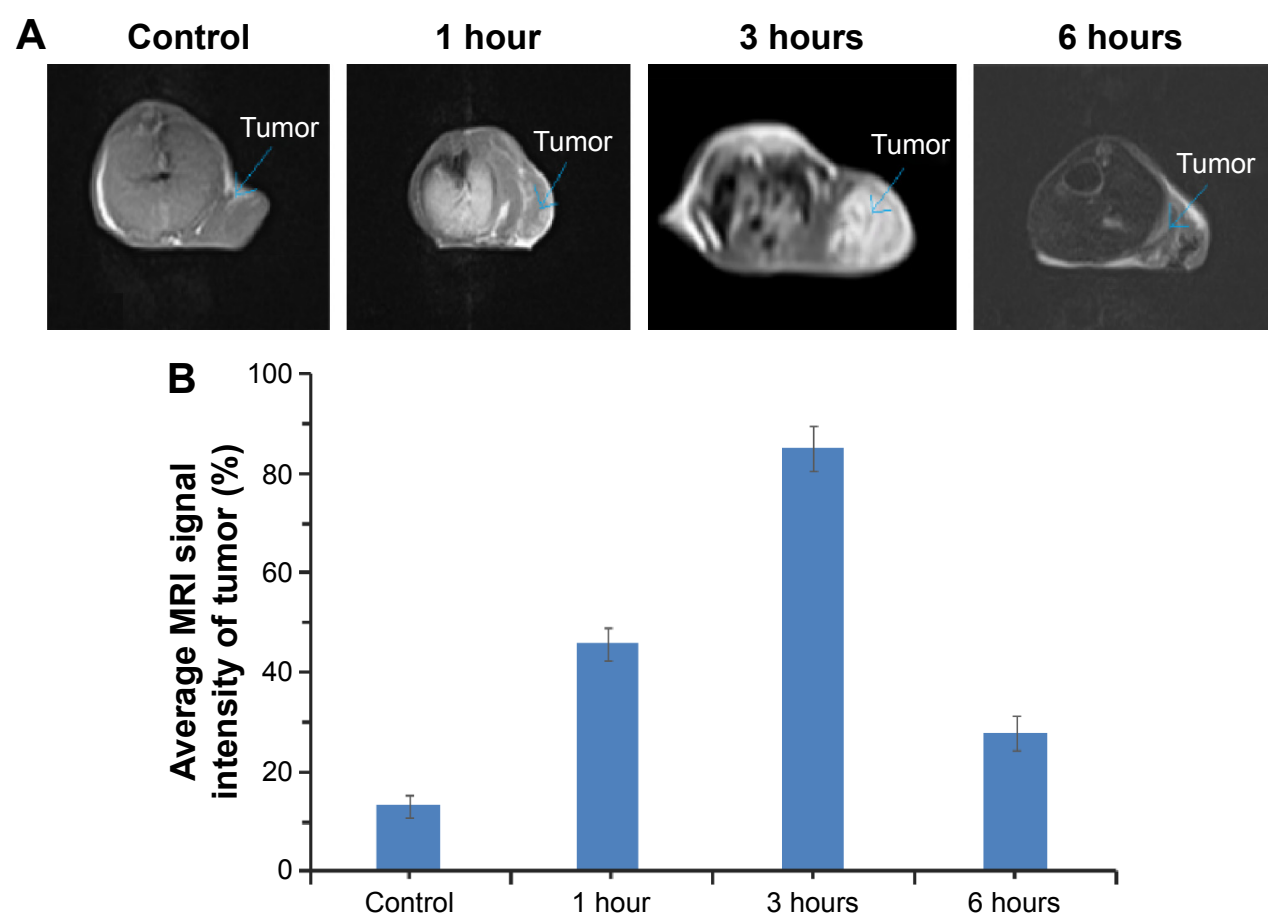

Figure 13 In vivo $M R I$ and the signal intensity.

Notes: (A) In vivo $\mathrm{T}_{1}$-weighted MRI; (B) the relative signal intensity in tumor after the administration of Gd-CDs/AFn (DOX)/FA nanoparticles. Data are presented as mean \pm standard deviation $(n=3)$.

Abbreviations: AFn, apoferritin; DOX, doxorubicin; FA, folic acid; Gd-CDs, gadolinium-carbon dots; MRI, magnetic resonance imaging.

\section{Acknowledgment}

This work was supported by grants from the National Natural Science Foundation of China (81503038).

\section{Disclosure}

The authors report no conflicts of interest in this work.

\section{References}

1. Stefanakis D, Philippidis A, Sygellou L, Filippidis G, Ghanotakis D. Synthesis of fluorescent carbon dots by a microwave heating process: structural characterization and cell imaging applications. J Nanopart Res. 2014;16(10):1-10.

2. Jahan S, Mansoor F, Naz S, Lei J, Kanwal S. Oxidative synthesis of highly fluorescent boron/nitrogen co-doped carbon nanodots enabling detection of photosensitizer and carcinogenic dye. Anal Chem. 2013; 85(21):10232-10239

3. Jie G, Zhang J, Jie G, Wang L. A novel quantum dot nanocluster as versatile probe for electrochemiluminescence and electrochemical assays of DNA and cancer cells. Biosens Bioelectron. 2014;52(4):69-75.

4. Wang Q, Huang X, Long Y, et al. Hollow luminescent carbon dots for drug delivery. Carbon N Y. 2013;9(4):192-199.

5. Zong J, Zhu Y, Yang X, Shen J, Li C. Synthesis of photoluminescent carbogenic dots using mesoporous silica spheres as nanoreactors. Chem Commun (Camb). 2011;47(2):764-766.

6. Cao L, Sahu S, Anilkumar P, et al. Carbon nanoparticles as visible-light photocatalysts for efficient $\mathrm{CO} 2$ conversion and beyond. $\mathrm{J} \mathrm{Am} \mathrm{Chem} \mathrm{Soc.}$ 2011;133(13):4754-4757.

7. Zong J, Yang XL, Trinchi A, et al. Photoluminescence enhancement of carbon dots by gold nanoparticles conjugated via PAMAM dendrimers. Nanoscale. 2013;5(22):11200-11206.
8. Tang J, Kong B, Wu H, et al. Carbon nanodots featuring efficient FRET for real-time monitoring of drug delivery and two-photon imaging. Adv Mater. 2013;25(45):6569-6574.

9. Mewada A, Pandey S, Thakur M, Jadhav D, Sharon M. Swarming carbon dots for folic acid mediated delivery of doxorubicin and biological imaging. J Mater Chem B. 2014;2(6):698-705.

10. Gong NH, Wang H, Li SH, et al. Microwave-assisted polyol synthesis of gadolinium-doped green luminescent carbon dots as a bimodal nanoprobe. Langmuir. 2014;30(36):10933-10939.

11. Shi L, Cao L, Gao R, et al. Synthesis and characterization of gadoliniumdoped nanotubular titania for enhanced photocatalysis. J Alloy Compd. 2014;617:756-762.

12. Chen $\mathrm{F}, \mathrm{Bu} \mathrm{W}$, Zhang $\mathrm{S}$, et al. Gd3+ ion-doped upconversion nanoprobes: relaxivity mechanism probing and sensitivity optimization. Adv Funct Mater. 2013;23(3):298-307.

13. Geraldes CF, Laurent S. Classification and basic properties of contrast agents for magnetic resonance imaging. Contrast Medla Mol Imaging. 2009;4(1):1-23.

14. Molino NM, Wang SW. Caged protein nanoparticles for drug delivery. Curr Opin Biotechnol. 2014;28(8):75-82.

15. Bellini M, Mazzucchelli S, Galbiati E, et al. Protein nanocages for self-triggered nuclear delivery of DNA-targeted chemotherapeutics in cancer cells. J Control Release. 2014;196:184-196.

16. Sanchez P, Valero E, Gálvez N, et al. MRI relaxation properties of water-soluble apoferritin-encapsulated gadolinium oxide-hydroxide nanoparticles. Dalton Trans. 2009;5(5):800-804.

17. Ji T, Zhao Y, Wang J, et al. Tumor fibroblast specific activation of a hybrid ferritin nanocage-based optical probe for tumor microenvironment imaging. Small. 2013;9(14):2427-2431.

18. Lin X, Xie J, Niu G, et al. Chimeric ferritin nanocages for multiple function loading and multimodal imaging. Nano Lett. 2011;11(2):814-819.

19. Blazkova I, Nguyen HV, Dostalova S, et al. Apoferritin modified magnetic particles as doxorubicin carriers for anticancer drug delivery. Int J Mol Sci. 2013;14(7):13391-13402. 
20. Gálvez N, Fernandez B, Valero E, et al. Apoferritin as a nanoreactor for preparing metallic nanoparticles. C R Chim. 2008;11(10):1207-1212.

21. Zhou L, Li Z, Liu Z, Ren J, Qu X. Luminescent carbon dot-gated nanovehicles for $\mathrm{pH}$-triggered intracellular controlled release and imaging. Langmuir. 2013;29(21):6396-6403.

22. Gumulec J, Fojtu M, Raudenska M, et al. Modulation of induced cytotoxicity of doxorubicin by using apoferritin and liposomal cages. Int J Mol Sci. 2014;15(12):22960-22977.

23. Kang HJ, Kang YJ, Lee YM, Shin HH, Chung SJ, Kang S. Developing an antibody-binding protein cage as a molecular recognition drug modular nanoplatform. Biomaterials. 2012;33(21):5423-5430.

24. Toole BP, Wight TN, Tammi MI. Hyaluronan-cell interactions in cancer and vascular disease. J Biol Chem. 2002;277(7):4593-4596.

25. Huang HC, Barua S, Sharma G, Dey SK, Rege K. Inorganic nanoparticles for cancer imaging and therapy. J Control Release. 2011;155(3): 344-357.

26. Hao R, Xing R, Xu Z, Hou Y, Gao S, Sun S. Synthesis, functionalization, and biomedical applications of multifunctional magnetic nanoparticles. Adv Mater. 2010;22(25):2729-2742.

27. Xiao N, Gu W, Wang H, Deng YX, She X, Ye L. T1-T2 dual-modal MRI of brain gliomas using PEGylated Gd-doped iron oxide nanoparticles. J Colloid Interface Sci. 2014;417(3):159-165.

28. Salinas-Castillo A, Ariza-Avidad M, Pritz C, et al. Carbon dots for copper detection with down and upconversion fluorescent properties as excitation sources. Chem Commun (Camb). 2013;49(11):1103-1105.
29. Sun H, Cao L, Lu L. Magnetite/reduced graphene oxide nanocomposites: one step solvothermal synthesis and use as a novel platform for removal of dye pollutants. Nano Res. 2011;4(6):550-562.

30. Shi J, Yu X, Wang L, et al. PEGylated fullerene/iron oxide nanocomposites for photodynamic therapy, targeted drug delivery and MR imaging. Biomaterials. 2013;34(37):9666-9677.

31. Zhang Z, Jia J, Lai Y, Ma Y, Weng J, Sun L. Conjugating folic acid to gold nanoparticles through glutathione for targeting and detecting cancer cells. Bioorg Med Chem. 2010;18(15):5528-5534.

32. Hou Z, Zhan C, Jiang Q, et al. Both FA- and mPEG-conjugated chitosan nanoparticles for targeted cellular uptake and enhanced tumor tissue distribution. Nanoscale Res Lett. 2011;6(40):563.

33. Sitharaman B, Wilson LJ. Gadonanotubes as new high-performance MRI contrast agents. Int J Nanomedicine. 2006;1(3):291-295.

34. Mogilireddy V, Déchamps-Olivier I, Alric C, et al. Thermodynamic stability and kinetic inertness of a Gd-DTPA bisamide complex grafted onto gold nanoparticles. Contrast Media Mol Imaging. 2014;10(3): 179-187.

35. Liang MM, Fan KL, Zhou M, et al. H-ferritin-nanocaged doxorubicin nanoparticles specifically target and kill tumors with a single-dose injection. Proc Natl Acad Sci U S A. 2014;111(41):14900-14905.
International Journal of Nanomedicine

\section{Publish your work in this journal}

The International Journal of Nanomedicine is an international, peerreviewed journal focusing on the application of nanotechnology in diagnostics, therapeutics, and drug delivery systems throughout the biomedical field. This journal is indexed on PubMed Central, MedLine, CAS, SciSearch $®$, Current Contents ${ }^{\circledR} /$ Clinical Medicine,

\section{Dovepress}

Journal Citation Reports/Science Edition, EMBase, Scopus and the Elsevier Bibliographic databases. The manuscript management system is completely online and includes a very quick and fair peer-review system, which is all easy to use. Visit http://www.dovepress.com/ testimonials.php to read real quotes from published authors. 